\title{
Effect of Allelopathic Potential of Corn, Sunflower, Field Capacity and Ascorbic Acid in Growth of Two Wheat cultivars
}

\author{
M. S. Faysal \\ Department of Biology, College of Education for Pure Science, University of Mosul, Mosul, Iraq \\ Email: $\underline{\text { dr.Mohmmadsf@gmail.com }}$
}

(Received December 16, 2019; Accepted February 05, 2020; Available online June 01, 2020)

DOI: 10.33899/edusj.2020.126418.1034, (C) 2020, College of Education for Pure Science, University of Mosul.

This is an open access article under the CC BY 4.0 license (http://creativecommons.org/licenses/by/4.0/).

\begin{abstract}
This study was carried out inside a wired house and included the planting two cultivars of wheat (Sham 6 - IPA 99) in soils containing roots residues of corn (Zea may L) and sunflower (Helianthns annuus $L$ ) with exposing plants to three levels of field capacity $(35,60,85 \%$ ) and spraying plants with three concentrations of ascorbic acid $(0,100,200) \mathrm{ppm}$ in order to know the effect of these factors on some physiological, biochemical and allelopathic potential of wheat. The experiment has been designed on the basis that it is factorial and over completely Randomized Design (C.R.D) Duncan's multiple range test at 5\% level was used to compare between the means and the results showed.

The addition of corn residues and sunflower showed a great effect in lowering plant height, relative water content, chlorophyll, number of grains / spike and grains yield with the increasing the concentration of catalase.

Exposure of plants to drought (35\% field capacity) showed a negative effect on all traits above comparing to field capacity $(60,85 \%)$ except for the increase in antioxidant enzymes (Peroxidase, Catalase).

Plants submitted under two types of stress (drought + allelopathy) can improve their growth and reduce the negative effect of drought and the inhibitory effect of residues by treating it with ascorbic acid especially in the concentration $200 \mathrm{ppm}$.

More over, sham 6 was significantly superior on IPA 99 in most physiological and biochemical characters, which was reflected in the increasing grains yield.
\end{abstract}

Keywords: allelopathy, Field capacity, Ascorbic acid, wheat.

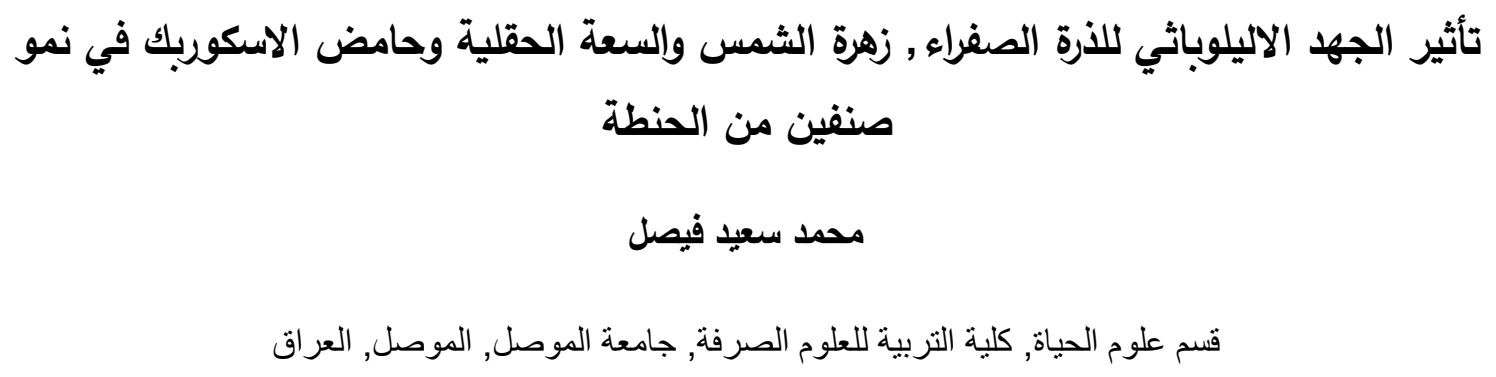


اجريت هذه الدراسة في البيت السلكي التابع لقسم علوم الحياة/ كلية الترببة للعلوم الصرفة وتضدنت زراعة صنفين من الحنطة (شام 6 وإباء 99) في ترب تحتوي متبقيات جذور الذرة الصفراء وزهرة الثمس مع تعريض النباتات إلى ثلاث مستويات من السعة الحقلية (35, 60, 85\%) ورش النباتات بثلاث تراكيز من حامض الاسكوربيك (صفر , 100, 200) جزء في المليون, وذلك لمعرفة تأثير تلك العوامل على بعض الصفات الفسلجية والبايوكيميائية والجهد الاليلوباثي للحنطة, صمدت التجربة على التئي

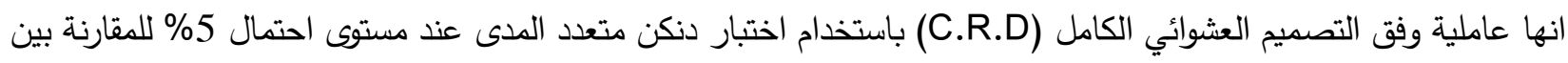
المتوسطات ويمكن ايجاز النتائج بما يلي:

اضافة متبقيات زهرة الثمس والذرة الصفراء اظهرت فاعلية كبيرة في خفض ارتفاع النبات والتهات والمساحة الورقية ومحتوى الماء النسبي والكلوروفيل الكلي وعدد الحبوب في السنبلة وحاصل الحبوب مع حصول زيادة في تركيز انزيم الكاتليز . تعريض النباتات إلى الجفاف (35\% سعة حقلية) اظهر تأثيراً سلبياً على جميع الصفات أعلاه مقارنة مع السعة الحقلية

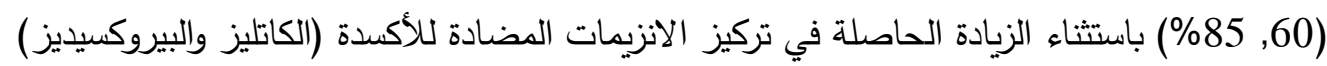

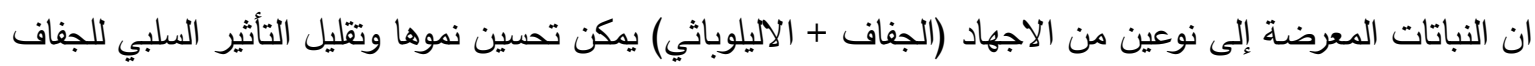

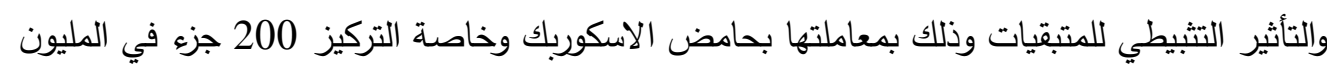

من جانب آخر تفوق صنف الحنطة شام 6 معنويا على الصنف اباء 99 في أغلب الصفات الفسلجية والبايوكيميائية والذي انعكس على زيادة حاصل الحبوب. الكلمات المفتاحية: الاليلوباثي - السعة الحقلية - حامض حضل الاسكوبك الاسكوبك - الحنطة

\section{Introduction}

Wheat is the first cultivated grain in the world wide in Iraq in terms of the importance of nutritional, taxonomy, it's economic, cultivated areas and production. In Iraq, the cultivated area of wheat was estimated at 7146,000 dunams in winter season 2017 [1].

The process of growing any plant needs essential elements. Water is most important element for all physiological activities,it affects the process of photosynthesis and the transfer of all compounds, including plant hormones, which is done only in the presence of water. Therefore exposing the plant to drought causes obstruction of many physiological processes and this is negatively reflected on the vegetative growth and the yield [2].

The study of Khaled (2010) [3] is about the impact of four levels of field capacity (100, 75, $50,25 \%)$ in the growth of wheat, the plant height has reduced with the reducing of the field capacity.

In addition to the impact of drought as an environmental factor, there are invisible factors that also affect negatively or positively the growth and productivity of plants, This a phenomenon is called direct or indirect effect, of substances produced by plants or residues in other plants through the secretion of allelochemicals in the environment is allelopathy [4].

A study about the allelopathy effects of wheat, corn and sunflower residues on growth and yield of wheat, revealed that the control treatment is significantly surpassed in all characters, residues of wheat cause the highest reduction[5].

According to Shao et al. (2019) [6] wheat has acquired two kinds of response system to drought pressure. The first one is that wheat can change its growth and developmental phenotype to deal with an arid environment through long ecological adaption, which is more linked with allelopathic regulation.

The seconed one is anti - drought mechanism, it maintains water balance by closing leaves stoma and decreasing water loss due to transpiration effect. 
On the other hand, Vitamins are one of the things that can be used to reduce environmental stresses, especially drought, they perform various biochemical functions, some of which are hormones such as growth regulators of cells and others act as antioxidants, such as vitamin $\mathrm{C}$ (Ascorbic), which is a non- enzymatic antioxidants that is exploited by plants under stress conditions [7]. Some researchers note that ascorbic acid (ASA) has a similar effect as a growth regulator that encourages growth by promoting photosynthesis and a relationship between leaf area it is increase in vegetative growth and plant content of ascorbic acid $[8,9]$.

The aim of this research was to get benefit from interaction concentrations of Ascorbic acid in reducing the visible environmental stress (drought) and non- visible (Allelopathy) on the growth of two wheat cultivars.

\section{Materials and Methods}

The study was conducted in the wired house and laboratories of the Department of Biology , College of Education for Pure Science, Mosul University in the season of 2018-2019. The soil used in this study is taken from the agricultural fields of the college of Agriculture and Forestry at depth (0-30) $\mathrm{cm}$. The soil was dried and passed through $2 \mathrm{~mm}$ diameter sieve. The plants of sunflower and corn were collected from the agricultural fields after the harvest they were brought to the laboratory and the root was separated from the vegetative. The root was well washed, then residues of the root was scraped onto a piece of nylon and left in the wire house to dry. The dried root is cut into small pieces and placed in an oven at $(70)^{\circ} \mathrm{C}$ for 48 hours after that is it and grinded with an electric mill and kept in bags under laboratory conditions to be used in later studies. $3 \mathrm{~g}$ of residue / $100 \mathrm{gm}$ soil were added to each pot that contains $4 \mathrm{Kg}$ of soil a liter of water was added to each pot. These pots were covered with a piece of cellophane perforated for ventilation and left in a wired house randomly fore a period of incubation for three weeks.

On 15Decembr2018, ten seeds of two cultivars of wheat (sham -6- and IPA -99-) were sown. The two cultivars were obtained from the examination and certification of seeds / Nineveh. After two weeks, seedings were reduced to five per each pot. After 40 days of sowing the plants were exposed to three levels of field capacity $(85,60,35 \%)$ by weighing each pot daily, plants were sprayed with $(0,100,200)$ ppm of ascorbic acid and control plant was sprayed with distilled water. After 52 days from the date of spraying 3 replicates were used for each treatment to study some physiological and biochemical characteristics. Three replicates were left in order to obtain the yield, the harvest data was on 20 May2019.

\section{Growth Parameters}

1- Plant height $(\mathrm{cm})$.

2- Leaf area was measured [10].

3- Relative water content [11].

4- Chlorophyll and carotenoid content of leaves were measured spectrophotometrically according to Lichtenthaler (1987) [12].

5- Assays for catalase activity (CAT) in the extract by the method Goth (2009) [13], absorbance was recorded $405 \mathrm{~nm}$ with a spectrophotometer.

6- Assays for peroxidase (POD) in the extract by the method according Kim and Yoo (1996) [14], absorbance was recorded $470 \mathrm{~nm}$ with a spectrophotometer.

7- Number of grains/ spike.

8- Grain yield.

The experiment is factorial in a completely randomized design (C.R.D) with four factors in six replications Duncan's multiple range test at 5\% level was used to compare between the means. The results have been analyzed by using the SAS program. 


\section{Results and Discussion Plant Height}

Table (1) illustrated a significant decrease in plant height at the probability $5 \%$ due to the effect of soil moisture levels $(35-60-85 \%)$ of field capacity by $(6.0,15.58 \%)$ sequentially compared to $85 \%$. Plant reduction can be attributed to water stress reduce that the relative water content that determines cell division and expansion and that expansion is more effective than division [15]. This result agreed with Al-Temimi et al. (2013) [16] since water deficit stress significantly reduced plant height, $50 \%$ field capacity treatment gave the highest values of plant height and leaf area of wheat compared to $(25,15 \%)$ field capacity. The results also showed a significant decrease due to the addition of sunflower and corn residues $(14.4,12.7 \%)$ compared with control treatment (without residues). These are consistent with Mohammed and Murshid (2018) [5] obtaining significant differences on the growth of wheat caused by the residues of wheat, corn and sunflower, ultimatly this was attributed to the allelopathic effect of plant residues on the division and elongation that influencing plant height. when the concentration of ascorbic acid to increased the height of the plant, as the concentration of $200 \mathrm{ppm}$ gave the highest average $50.9 \mathrm{~cm}$ and superiority rate 10.39 compared with control.

The plant height of spray plants with ascorbic acid is increased which means the ascorbic, plays multiple roles in plant growth such as cell division, and cell wall expansion[8].

This is confirmed by Abdel Adeem and Ahmed (2017) [17] the spraying of corn plant with ASA in concentration $200 \mathrm{ppm}$ increased the plant height of compared to the control.

The interaction effect between residues and ascorbic has a significant increase in plant height at 200 ppm concentration in control compared with other treatments.

With respect to the combined effect (cultivar $\times$ residues $\times$ field capacity) the sham -6 - was superior in treatment with no residues and at field capacity $85 \%$. The results of interaction (cultivar $\times$ residues $\times$ field capacity $\times$ ascorbic) showed the highest value in sham 6 of field capacity $85 \%$ at 200 ppm concentration of ascorbic acid.

\section{Leaf area}

The leaf is the main member of plant that gets the most effective photosynthesis. Table (2) exhibited that the field capacity $85 \%$ gave the highest leaf area $(17.5) \mathrm{cm}^{2}$. while the addition of irrigation water of the field capacity $35 \%$ less values $(12.2) \mathrm{cm}^{2}$. This means that water stress reduced leaf area, and also the received light was reduced, this negatively effects the synthesis of organic acid. The results agree with Almahasneh (2012) [18] that leaf area of wheat was significantly affected by water deficit, it returns to the decrease of flag leaf area subject to water stress led to the reduced water absorption. This is reflected in the reduced water potential, which causes a decrease in energy production in light reaction [19]. On the other hand, the leaf area increased with increasing concentration of ascorbic acid, the best treatment was recorded when spraying plants with a $200 \mathrm{ppm}$ compared the treatment $100 \mathrm{ppm}$ and control, the percentage of increase was $(9.2,24.1 \%)$ respectively. The results were consistent with Ali et al. (2015) [20] They found an increase in corn leaf area when treated with ascorbic acid because the acid participates in a variety of processes including photosynthesis, cell wall growth, cell expansion and synthesis of ethylene and gibberellin [21]. The results showed an inhibition effects when adding sunflower and corn residues, and corn showed a higher allelopathic effect than sunflower residues compared to control. The results agree with Morshed and Mohammed (2016) [22] that found the control treatment gave the highest value for the leaf area, the residues of wheat, corn and sunflower caused a significant reduction in leaf area. This may be due to residues that were at high levels, which increase the concentration of allelopathic compounds, especially mono phenols derived interfere to some degree with many vital plant processes, including cell division, water balance and phytohormone activity which leads to stop the work plant growth regulator especially auxin and 
cytokines resulting in reduced leaf area [23, 24]. With regard to the interaction between cultivars and field capacity, sham -6- was superior to the field capacity $85 \%$. With respect to the residues and field capacity treatment with no residues was outperformed at field capacity $85 \%$.

In terms of interaction (cultivar $\times$ field capacity $\times$ ascorbic) there was a significant superiority of sham 6 at field capacity $85 \%$ and asorbic acid concentration $200 \mathrm{ppm}$.

\section{Relative Water Content}

Table (3) indicates that the drought caused a negative effect on the characteristics of relative water content, where has a significant decline when field capacity $35 \%$ compared to $60 \%, 85 \%$ the rate of decline $(34.8,14.2 \%)$ respectively. This agrees with Hussein and Khursheed (2014) [25] the significant decrease in water content of the wheat leaves with increasing water deficiency in soil, the reduction probably due to stomata closure, reducing water absorption and transpiration rate which reduce the $\mathrm{CO}_{2} / \mathrm{O}_{2}$ ratio in leaves and inhibits photosynthesis, these conditions increase the rate of reactive oxygen species (ROS). Table (3) also shows an inhibition due to the addition of corn and sunflower residues compared to the control treatment the rate of decline was $(12.0,18.5 \%)$ respectively. Our results are consistent with Ali et al.(2005) [26] indicating that soils contain $2 \%$ powder chard leave led to a significant

Table (1) Effect of corn, sunflower residues and field capacity in the plant height $(\mathrm{cm})$ of wheat treated with ascorbic acid

\begin{tabular}{|c|c|c|c|c|c|c|c|c|c|c|c|c|}
\hline \multirow[b]{2}{*}{ Cultivar } & \multirow[b]{2}{*}{ Residues } & \multirow{2}{*}{$\begin{array}{l}\text { Ascorbic } \\
\text { acid }\end{array}$} & \multicolumn{3}{|c|}{ Field Capacity } & \multirow{2}{*}{$\begin{array}{c}\text { Cultivar } \\
\times \\
\text { Residues } \\
\times \\
\text { Ascorbic }\end{array}$} & \multirow{2}{*}{$\begin{array}{c}\text { Cultivar } \\
\times \\
\text { Residues }\end{array}$} & \multirow{2}{*}{$\begin{array}{c}\text { Cultivar } \\
\times \\
\text { Ascorbic }\end{array}$} & \multirow{2}{*}{$\begin{array}{c}\text { Residues } \\
\times \\
\text { Ascorbic }\end{array}$} & \multicolumn{3}{|c|}{$\begin{array}{c}\text { Cultivar } \times \text { Residues } \times \text { Field } \\
\text { Capacity }\end{array}$} \\
\hline & & & 85 & 60 & 35 & & & & & 85 & 60 & 35 \\
\hline \multirow{9}{*}{ IPA 99} & \multirow{3}{*}{ Control } & 0 & $\begin{array}{c}* 50.201 \\
\mathrm{~g}-\mathrm{i}\end{array}$ & $\begin{array}{c}47.200 \\
\mathrm{k}-\mathrm{s}\end{array}$ & $\begin{array}{c}46.40 \\
\mathrm{~m}-\mathrm{t}\end{array}$ & $\begin{array}{c}47.933 \\
\text { fg }\end{array}$ & \multirow{3}{*}{$\begin{array}{c}48.633 \\
b\end{array}$} & $\begin{array}{c}46.233 \\
\mathrm{~d}\end{array}$ & $\begin{array}{c}51.150 \\
\mathrm{c}\end{array}$ & $\begin{array}{c}51.666 \\
c\end{array}$ & $\begin{array}{c}48.233 \\
\mathrm{de}\end{array}$ & $\begin{array}{l}46.000 \\
\mathrm{f}\end{array}$ \\
\hline & & 100 & $\begin{array}{c}50.30 \\
\text { g-k }\end{array}$ & $\begin{array}{c}47.400 \\
\mathrm{j}-\mathrm{r}\end{array}$ & $\begin{array}{c}46.500 \\
\text { i-t }\end{array}$ & $\begin{array}{c}48.066 \\
e-j\end{array}$ & & $\begin{array}{c}45.744 \\
\mathrm{~d}\end{array}$ & $\begin{array}{c}53.183 \\
\mathrm{~b}\end{array}$ & & & \\
\hline & & 200 & $\begin{array}{c}54.500 \\
\text { ef }\end{array}$ & $\begin{array}{c}50.100 \\
\text { g-m }\end{array}$ & $\begin{array}{c}45.100 \\
\text { o-t }\end{array}$ & $\begin{array}{c}49.900 \\
\mathrm{de}\end{array}$ & & $\begin{array}{c}49.022 \\
\mathrm{c}\end{array}$ & $\begin{array}{c}55.650 \\
\mathrm{a}\end{array}$ & & & \\
\hline & \multirow{3}{*}{ Corn } & 0 & $\begin{array}{c}48.100 \\
\text { i-o }\end{array}$ & $\begin{array}{c}45.300 \\
\mathrm{o}-\mathrm{u}\end{array}$ & $\begin{array}{c}42.000 \\
\mathrm{u}-\mathrm{w}\end{array}$ & $\begin{array}{c}45.133 \\
\mathrm{i}\end{array}$ & \multirow{3}{*}{$\begin{array}{c}46.122 \\
\text { de }\end{array}$} & & $\begin{array}{c}43.233 \\
\mathrm{f}\end{array}$ & $\begin{array}{c}48.166 \\
\text { de }\end{array}$ & $\begin{array}{c}46.737 \\
\text { ef }\end{array}$ & $\begin{array}{c}43.400 \\
\mathrm{gi}\end{array}$ \\
\hline & & 100 & $\begin{array}{c}43.300 \\
t-v\end{array}$ & $\begin{array}{c}43.600 \\
s-v\end{array}$ & $\begin{array}{c}42.100 \\
\mathrm{u}-\mathrm{w}\end{array}$ & $\begin{array}{c}43.000 \\
\mathrm{j}\end{array}$ & & & $\begin{array}{c}44.850 \\
\mathrm{e}\end{array}$ & & & \\
\hline & & 200 & $\begin{array}{c}53.100 \\
\text { e-h }\end{array}$ & $\begin{array}{c}51.300 \\
\mathrm{f}-\mathrm{i}\end{array}$ & $\begin{array}{c}46.300 \\
n-t\end{array}$ & $\begin{array}{c}50.233 \\
\mathrm{~d}\end{array}$ & & & $\begin{array}{c}48.750 \\
d\end{array}$ & & & \\
\hline & \multirow{3}{*}{ Sunflower } & 0 & $\begin{array}{c}53.700 \\
\text { e-g }\end{array}$ & $\begin{array}{c}44.200 \\
q-v\end{array}$ & $\begin{array}{c}39.000 \\
\text { wx }\end{array}$ & $\begin{array}{c}45.633 \\
\mathrm{hi}\end{array}$ & \multirow{3}{*}{$\begin{array}{c}46.244 \\
\quad \mathrm{~d}\end{array}$} & & $\begin{array}{c}44.183 \\
\text { ef }\end{array}$ & $\begin{array}{l}49.900 \\
\mathrm{~cd}\end{array}$ & $\begin{array}{l}45.900 \\
\mathrm{f}\end{array}$ & $\begin{array}{c}42.933 \\
\mathrm{~g}\end{array}$ \\
\hline & & 100 & $\begin{array}{c}47.500 \\
\mathrm{j}-\mathrm{q}\end{array}$ & $\begin{array}{c}46.500 \\
\text { i-t }\end{array}$ & $\begin{array}{c}44.500 \\
\text { p-v }\end{array}$ & $\begin{array}{c}46.166 \\
n-j\end{array}$ & & & $\begin{array}{c}47.616 \\
d\end{array}$ & & & \\
\hline & & 200 & $\begin{array}{c}48.500 \\
\text { i-o }\end{array}$ & $\begin{array}{c}47.000 \\
k-t\end{array}$ & $\begin{array}{c}45.300 \\
\mathrm{o}-\mathrm{t}\end{array}$ & $\begin{array}{c}46.933 \\
\mathrm{~h}-\mathrm{j}\end{array}$ & & & $\begin{array}{c}48.566 \\
d\end{array}$ & & & \\
\hline \multirow{10}{*}{ Sham 6} & \multirow{3}{*}{ Control } & 0 & $\begin{array}{c}58.500 \\
\mathrm{~cd}\end{array}$ & $\begin{array}{c}56.100 \\
c-e\end{array}$ & $\begin{array}{c}48.500 \\
\text { i-o }\end{array}$ & $\begin{array}{c}54.316 \\
\mathrm{c}\end{array}$ & \multirow{3}{*}{$\begin{array}{c}58.022 \\
\mathrm{a}\end{array}$} & $\begin{array}{c}46.144 \\
\mathrm{~d}\end{array}$ & & $\begin{array}{c}63.500 \\
\mathrm{a}\end{array}$ & $\begin{array}{c}59.200 \\
\mathrm{~b}\end{array}$ & $\begin{array}{c}51.366 \\
\mathrm{c}\end{array}$ \\
\hline & & 100 & $\begin{array}{c}65.000 \\
\mathrm{a} \\
\end{array}$ & $\begin{array}{c}59.200 \\
\mathrm{c}\end{array}$ & $\begin{array}{c}50.200 \\
\mathrm{~g}-\mathrm{i}\end{array}$ & $\begin{array}{c}58.300 \\
\mathrm{~b}\end{array}$ & & $\begin{array}{c}51.355 \\
\mathrm{~b}\end{array}$ & & & & \\
\hline & & 200 & $\begin{array}{c}66.500 \\
\mathrm{a}\end{array}$ & $\begin{array}{c}62.300 \\
\mathrm{~b}\end{array}$ & $\begin{array}{c}55.400 \\
\text { de }\end{array}$ & $\begin{array}{c}61.400 \\
\mathrm{a}\end{array}$ & & $\begin{array}{c}52.955 \\
\mathrm{a}\end{array}$ & & & & \\
\hline & \multirow{3}{*}{ Corn } & 0 & $\begin{array}{c}43.500 \\
\text { s-t }\end{array}$ & $\begin{array}{c}41.200 \\
\mathrm{~V}-\mathrm{X}\end{array}$ & $\begin{array}{c}39.300 \\
\text { wx }\end{array}$ & $\begin{array}{c}41.330 \\
\mathrm{j}\end{array}$ & \multirow{3}{*}{$\begin{array}{c}45.100 \\
\mathrm{e}\end{array}$} & & & $\begin{array}{c}48.266 \\
\text { de }\end{array}$ & $\begin{array}{c}46.366 \\
\text { ef }\end{array}$ & $\begin{array}{c}40.666 \\
n\end{array}$ \\
\hline & & 100 & $\begin{array}{c}50.300 \\
g-j\end{array}$ & $\begin{array}{c}48.400 \\
\text { i-o }\end{array}$ & $\begin{array}{l}41.400 \\
\mathrm{v}-\mathrm{x}\end{array}$ & $\begin{array}{c}46.700 \\
\text { g-i }\end{array}$ & & & & & & \\
\hline & & 200 & $\begin{array}{c}51.000 \\
f-j\end{array}$ & $\begin{array}{c}49.500 \\
\text { h-n }\end{array}$ & $\begin{array}{c}41.300 \\
\text { v-x }\end{array}$ & $\begin{array}{c}47.266 \\
\text { f-h }\end{array}$ & & & & & & \\
\hline & \multirow{3}{*}{ Sunflower } & 0 & $\begin{array}{c}46.200 \\
n-t\end{array}$ & $\begin{array}{c}43.70 \\
\mathrm{r}-\mathrm{V}\end{array}$ & $\begin{array}{c}38.300 \\
\mathrm{x} \\
\end{array}$ & $\begin{array}{c}42.733 \\
j\end{array}$ & \multirow{3}{*}{$\begin{array}{c}47.33 \\
c\end{array}$} & & & $\begin{array}{c}51.600 \\
\mathrm{c}\end{array}$ & $\begin{array}{c}47.866 \\
\text { ef }\end{array}$ & $\begin{array}{c}42.533 \\
\mathrm{~g}\end{array}$ \\
\hline & & 100 & $\begin{array}{c}53.500 \\
e-g\end{array}$ & $\begin{array}{c}45.200 \\
\mathrm{o}-\mathrm{u}\end{array}$ & $\begin{array}{c}45.20 \\
0-\mathrm{t}\end{array}$ & $\begin{array}{c}49.066 \\
\text { d-f }\end{array}$ & & & & & & \\
\hline & & 200 & $\begin{array}{c}55.100 \\
\mathrm{e}\end{array}$ & $\begin{array}{c}51.400 \\
\mathrm{f}-\mathrm{i}\end{array}$ & $\begin{array}{c}44.100 \\
q-v\end{array}$ & $\begin{array}{c}50.200 \\
\mathrm{~d}\end{array}$ & & & & & & \\
\hline & & & & & & & Cultivar & Residues & Ascorbic & Residu & $\begin{array}{l}\times \text { Field C } \\
\text { Aserobi }\end{array}$ & $\operatorname{acity} x$ \\
\hline $\begin{array}{c}\text { Cultivar } \\
\times\end{array}$ & \multicolumn{2}{|c|}{ IPA } & $\begin{array}{c}49.911 \\
c\end{array}$ & $\begin{array}{c}46.955 \\
\mathrm{~d}\end{array}$ & $\begin{array}{c}44.133 \\
\mathrm{e}\end{array}$ & & $\begin{array}{c}47.00 \\
\mathrm{~b}\end{array}$ & & & & & \\
\hline
\end{tabular}


Journal of Education and Science (ISSN 1812-125X), Vol: 29, No: 2, 2020 (260-278)

\begin{tabular}{|c|c|c|c|c|c|c|c|c|c|c|c|}
\hline \multicolumn{2}{|c|}{$\begin{array}{c}\text { Field } \\
\text { Capacity }\end{array}$} & Sham 6 & $\begin{array}{c}54.455 \\
\mathrm{a} \\
\end{array}$ & $\begin{array}{c}51.144 \\
\mathrm{~b}\end{array}$ & $\begin{array}{c}44.855 \\
\mathrm{e}\end{array}$ & $\begin{array}{c}50.15 \\
\mathrm{a} \\
\end{array}$ & & & & & \\
\hline \multirow{3}{*}{\multicolumn{2}{|c|}{$\begin{array}{c}\text { Residues } \\
\times \\
\text { Field } \\
\text { Capacity }\end{array}$}} & Con-T & $\begin{array}{c}57.583 \\
\mathrm{a}\end{array}$ & $\begin{array}{c}53.716 \\
\text { b }\end{array}$ & $\begin{array}{c}48.683 \\
d\end{array}$ & & $\begin{array}{c}53.327 \\
\mathrm{a}\end{array}$ & & & & \\
\hline & & Corn & $\begin{array}{c}48.21 \\
\text { bd }\end{array}$ & $\begin{array}{c}46.550 \\
\mathrm{e}\end{array}$ & $\begin{array}{c}42.066 \\
\text { f }\end{array}$ & & $\begin{array}{c}45.611 \\
\mathrm{c}\end{array}$ & & & & \\
\hline & & Sunflower & $\begin{array}{c}50.750 \\
\mathrm{c}\end{array}$ & $\begin{array}{c}46.883 \\
\mathrm{e}\end{array}$ & $\begin{array}{c}42.733 \\
\text { f }\end{array}$ & & $\begin{array}{c}46.788 \\
b\end{array}$ & & & & \\
\hline \multirow{3}{*}{\multicolumn{2}{|c|}{$\begin{array}{c}\text { Ascorbic } \\
\times \\
\text { Field } \\
\text { Capacity }\end{array}$}} & 0 & $\begin{array}{c}50.033 \\
\mathrm{c}\end{array}$ & $\begin{array}{c}46.283 \\
\mathrm{~d}\end{array}$ & $\begin{array}{c}42.250 \\
\mathrm{e}\end{array}$ & & & $\begin{array}{c}46.188 \\
\text { c }\end{array}$ & & & \\
\hline & & 100 & $\begin{array}{c}51.733 \\
\text { b }\end{array}$ & $\begin{array}{c}48.933 \\
\mathrm{c}\end{array}$ & $\begin{array}{c}44.983 \\
d\end{array}$ & & & $\begin{array}{c}48.550 \\
\text { b }\end{array}$ & & & \\
\hline & & 200 & $\begin{array}{c}54.783 \\
\mathrm{a}\end{array}$ & $\begin{array}{c}51.933 \\
\text { b }\end{array}$ & $\begin{array}{c}46.250 \\
d\end{array}$ & & & $\begin{array}{c}50.988 \\
\mathrm{a}\end{array}$ & & & \\
\hline \multirow{6}{*}{ 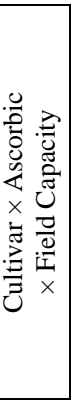 } & \multirow{3}{*}{ 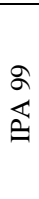 } & 0 & $\begin{array}{c}50.666 \\
\mathrm{~cd}\end{array}$ & $\begin{array}{c}45.566 \\
\text { ef }\end{array}$ & $\begin{array}{c}42.466 \\
\mathrm{~g}\end{array}$ & & \multirow{3}{*}{ Control } & 0 & $\begin{array}{c}45.350 \\
\mathrm{~cd} \\
\end{array}$ & $\begin{array}{c}51.650 \\
\text { e-g }\end{array}$ & $\begin{array}{c}47.450 \\
\mathrm{i}-\mathrm{k}\end{array}$ \\
\hline & & 100 & $\begin{array}{c}47.033 \\
\mathrm{e} \\
\end{array}$ & $\begin{array}{c}45.833 \\
\text { ef }\end{array}$ & $\begin{array}{c}44.366 \\
\mathrm{f}\end{array}$ & & & 100 & $\begin{array}{c}57.90 \\
\mathrm{~b}\end{array}$ & $\begin{array}{c}53.300 \\
\mathrm{de}\end{array}$ & $\begin{array}{c}48.500 \\
h-j\end{array}$ \\
\hline & & 200 & $\begin{array}{c}52.033 \\
\mathrm{c} \\
\end{array}$ & $\begin{array}{c}49.466 \\
\mathrm{~d}\end{array}$ & $\begin{array}{c}45.566 \\
\text { ef }\end{array}$ & & & 200 & $\begin{array}{c}60.500 \\
\mathrm{a} \\
\end{array}$ & $\begin{array}{c}56.200 \\
\mathrm{bc}\end{array}$ & $\begin{array}{c}50.250 \\
\mathrm{f}-\mathrm{h}\end{array}$ \\
\hline & \multirow{3}{*}{ 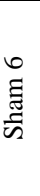 } & 0 & $\begin{array}{c}49.400 \\
\mathrm{~d}\end{array}$ & $\begin{array}{c}47.000 \\
\mathrm{e}\end{array}$ & $\begin{array}{c}42.033 \\
\mathrm{~g}\end{array}$ & & \multirow{3}{*}{ Corn } & 0 & $\begin{array}{c}45.800 \\
\mathrm{~km}\end{array}$ & $\begin{array}{c}43.250 \\
\text { no }\end{array}$ & $\begin{array}{c}40.650 \\
\mathrm{pq}\end{array}$ \\
\hline & & 100 & $\begin{array}{c}56.433 \\
\text { a }\end{array}$ & $\begin{array}{c}52.033 \\
\mathrm{c}\end{array}$ & $\begin{array}{c}45.600 \\
\text { ef }\end{array}$ & & & 100 & $\begin{array}{c}46.800 \\
\text { i- } 1\end{array}$ & $\begin{array}{c}46.000 \\
\mathrm{i}-1\end{array}$ & $\begin{array}{c}41.750 \\
\text { op }\end{array}$ \\
\hline & & 200 & $\begin{array}{c}57.533 \\
\mathrm{a} \\
\end{array}$ & $\begin{array}{c}54.400 \\
\mathrm{~b}\end{array}$ & $\begin{array}{c}46.933 \\
\mathrm{e}\end{array}$ & & & 200 & $\begin{array}{c}52.050 \\
\text { d-f }\end{array}$ & $\begin{array}{c}50.400 \\
\text { f-h }\end{array}$ & $\begin{array}{c}43.800 \\
\mathrm{~m}-\mathrm{o}\end{array}$ \\
\hline \multirow{3}{*}{\multicolumn{2}{|c|}{$\begin{array}{c}\text { Field } \\
\text { Capacity }\end{array}$}} & & \multirow{3}{*}{$\begin{array}{c}52.183 \\
\mathrm{a}\end{array}$} & \multirow{3}{*}{$\begin{array}{c}49.050 \\
\mathrm{~b}\end{array}$} & \multirow{3}{*}{$\begin{array}{c}44.494 \\
c\end{array}$} & & \multirow{3}{*}{ Sunflower } & 0 & $\begin{array}{c}49.950 \\
\text { f-h }\end{array}$ & $\begin{array}{c}43.950 \\
\text { m-o }\end{array}$ & $\begin{array}{c}38.650 \\
q\end{array}$ \\
\hline & & & & & & & & 100 & $\begin{array}{c}50.500 \\
\mathrm{f}-\mathrm{h} \\
\end{array}$ & $\begin{array}{c}47.500 \\
\mathrm{i}-\mathrm{k}\end{array}$ & $\begin{array}{c}44.850 \\
1-n\end{array}$ \\
\hline & & & & & & & & 200 & $\begin{array}{c}51.800 \\
\text { ef }\end{array}$ & $\begin{array}{c}49.200 \\
\text { g-i }\end{array}$ & $\begin{array}{c}44.700 \\
1-n\end{array}$ \\
\hline
\end{tabular}

* Means followed by different letters are a significantly at 0.05 level, Duncan's multiple range test.

Table (2) Effect of corn, sunflower residues and field capacity in leaf area $\left(\mathrm{cm}^{2}\right)$ of wheat treated with ascorbic acid

\begin{tabular}{|c|c|c|c|c|c|c|c|c|c|c|c|c|}
\hline \multirow[b]{2}{*}{ Cultivar } & \multirow[b]{2}{*}{ Residues } & \multirow[b]{2}{*}{$\begin{array}{c}\text { Ascorbic } \\
\text { acid }\end{array}$} & \multicolumn{3}{|c|}{ Field Capacity } & \multirow{2}{*}{$\begin{array}{c}\text { Cultivar } \\
\times \\
\text { Residues } \\
\times \\
\text { Ascorbic }\end{array}$} & \multirow{2}{*}{$\begin{array}{c}\text { Cultivar } \\
\times \\
\text { Residues }\end{array}$} & \multirow{2}{*}{$\begin{array}{c}\text { Cultivar } \\
\times \\
\text { Ascorbic }\end{array}$} & \multirow{2}{*}{$\begin{array}{c}\text { Residues } \\
\times \\
\text { Ascorbic }\end{array}$} & \multicolumn{3}{|c|}{$\begin{array}{c}\text { Cultivar } \times \text { Residues } \times \text { Field } \\
\text { Capacity }\end{array}$} \\
\hline & & & 85 & 60 & 35 & & & & & 85 & 60 & 35 \\
\hline \multirow{9}{*}{ IPA 99} & \multirow{3}{*}{ Control } & 0 & $\begin{array}{c}15.600 \\
\mathrm{e}-\mathrm{i}\end{array}$ & $\begin{array}{l}12.500 \\
\text { i-r }\end{array}$ & $\begin{array}{c}19.700 \\
\text { r-t }\end{array}$ & $\begin{array}{c}12.600 \\
\mathrm{~g}-\mathrm{i}\end{array}$ & \multirow{3}{*}{$\begin{array}{c}14.870 \\
\mathrm{c}\end{array}$} & $\begin{array}{c}12.014 \\
\mathrm{~d}\end{array}$ & $\begin{array}{c}14.616 \\
\mathrm{de}\end{array}$ & $\begin{array}{c}17.800 \\
\text { b }\end{array}$ & $\begin{array}{c}14.700 \\
\mathrm{de}\end{array}$ & $\begin{array}{c}12.111 \\
\mathrm{~g}\end{array}$ \\
\hline & & 100 & $\begin{array}{c}18.400 \\
\text { b-e }\end{array}$ & $\begin{array}{c}15.500 \\
\mathrm{e}-\mathrm{i}\end{array}$ & $\begin{array}{c}12.733 \\
\mathrm{i}-\mathrm{q}\end{array}$ & $\begin{array}{c}15.544 \\
\mathrm{~cd}\end{array}$ & & $\begin{array}{c}14.481 \\
\mathrm{c}\end{array}$ & $\begin{array}{c}16.088 \\
\mathrm{bc}\end{array}$ & & & \\
\hline & & 200 & $\begin{array}{c}19.400 \\
\mathrm{bc}\end{array}$ & $\begin{array}{c}16.100 \\
d-j\end{array}$ & $\begin{array}{c}13.900 \\
j-p\end{array}$ & $\begin{array}{c}16.466 \\
b c\end{array}$ & & $\begin{array}{c}15.548 \\
b\end{array}$ & $\begin{array}{c}17.666 \\
\mathrm{a}\end{array}$ & & & \\
\hline & \multirow{3}{*}{ Corn } & 0 & $\begin{array}{c}13.300 \\
j-p\end{array}$ & $\begin{array}{c}12.200 \\
\mathrm{~m}-\mathrm{t}\end{array}$ & $\begin{array}{c}9.300 \\
t\end{array}$ & $\begin{array}{c}11.600 \\
\mathrm{i}\end{array}$ & \multirow{3}{*}{$\begin{array}{c}12.996 \\
\mathrm{~d}\end{array}$} & & $\begin{array}{c}12.666 \\
\mathrm{f}\end{array}$ & $\begin{array}{c}14.966 \\
\text { de }\end{array}$ & $\begin{array}{c}13.722 \\
\text { ef }\end{array}$ & $\begin{array}{c}10.300 \\
\mathrm{~h}\end{array}$ \\
\hline & & 100 & $\begin{array}{c}15.500 \\
\mathrm{e}-\mathrm{i}\end{array}$ & $\begin{array}{c}13.700 \\
j-p\end{array}$ & $\begin{array}{c}10.200 \\
q-t\end{array}$ & $\begin{array}{c}13.133 \\
\mathrm{f}-\mathrm{i}\end{array}$ & & & $\begin{array}{c}13.966 \\
\mathrm{e}\end{array}$ & & & \\
\hline & & 200 & $\begin{array}{c}16.100 \\
d-j\end{array}$ & $\begin{array}{c}15.267 \\
\mathrm{e}-\mathrm{i}\end{array}$ & $\begin{array}{c}11.400 \\
0-t\end{array}$ & $\begin{array}{c}14.255 \\
\text { d-f }\end{array}$ & & & $\begin{array}{c}15.044 \\
\mathrm{c}-\mathrm{e}\end{array}$ & & & \\
\hline & \multirow{3}{*}{ Sunflower } & 0 & $\begin{array}{c}13.700 \\
j-p\end{array}$ & $\begin{array}{c}12.333 \\
\mathrm{~m}-\mathrm{s}\end{array}$ & $\begin{array}{c}9.500 \\
\text { St }\end{array}$ & $\begin{array}{c}11.844 \\
\mathrm{hi}\end{array}$ & \multirow{3}{*}{$\begin{array}{c}14.177 \\
\mathrm{c}\end{array}$} & & $\begin{array}{c}12.572 \\
\mathrm{f}\end{array}$ & $\begin{array}{c}16.622 \\
b c\end{array}$ & $\begin{array}{c}14.477 \\
\text { de }\end{array}$ & $\begin{array}{c}11.433 \\
\mathrm{gh}\end{array}$ \\
\hline & & 100 & $\begin{array}{c}17.300 \\
\text { b-h }\end{array}$ & $\begin{array}{c}15.100 \\
\mathrm{~g}-\mathrm{n}\end{array}$ & $\begin{array}{c}11.900 \\
\mathrm{o}-\mathrm{t}\end{array}$ & $\begin{array}{c}14.766 \\
\text { de }\end{array}$ & & & $\begin{array}{c}15.250 \\
\mathrm{~cd}\end{array}$ & & & \\
\hline & & 200 & $\begin{array}{c}18.867 \\
\text { b-d }\end{array}$ & $\begin{array}{c}16.000 \\
\mathrm{~d}-\mathrm{k}\end{array}$ & $\begin{array}{c}12.900 \\
\mathrm{k}-\mathrm{q}\end{array}$ & $\begin{array}{c}15.922 \\
\mathrm{~cd}\end{array}$ & & & $\begin{array}{c}16.766 \\
a b\end{array}$ & & & \\
\hline \multirow{6}{*}{ Sham 6} & \multirow{3}{*}{ Control } & 0 & $\begin{array}{c}19.500 \\
\mathrm{bc}\end{array}$ & $\begin{array}{c}16.700 \\
\mathrm{c}-\mathrm{i}\end{array}$ & $\begin{array}{c}13.700 \\
\mathrm{j}-\mathrm{p}\end{array}$ & $\begin{array}{c}16.633 \\
b c\end{array}$ & \multirow{3}{*}{17.377} & $\begin{array}{c}14.555 \\
\mathrm{c}\end{array}$ & & $\begin{array}{c}20.888 \\
\mathrm{a} \\
\end{array}$ & $\begin{array}{c}16.977 \\
\text { bc }\end{array}$ & $\begin{array}{c}14.266 \\
\mathrm{e}\end{array}$ \\
\hline & & 100 & $\begin{array}{c}19.900 \\
\mathrm{~b}\end{array}$ & $\begin{array}{c}16.100 \\
d-j\end{array}$ & $\begin{array}{c}13.900 \\
j-p\end{array}$ & $\begin{array}{c}16.633 \\
b c\end{array}$ & & $\begin{array}{c}15.722 \\
\mathrm{~b}\end{array}$ & & & & \\
\hline & & 200 & $\begin{array}{c}23.267 \\
\mathrm{a} \\
\end{array}$ & $\begin{array}{c}18.133 \\
\text { b-q }\end{array}$ & $\begin{array}{c}15.200 \\
\text { g-n }\end{array}$ & $\begin{array}{c}18.866 \\
\mathrm{a} \\
\end{array}$ & & $\begin{array}{c}17.437 \\
\mathrm{a} \\
\end{array}$ & & & & \\
\hline & \multirow{3}{*}{ Corn } & 0 & $\begin{array}{c}15.100 \\
\text { g-n }\end{array}$ & $\begin{array}{c}13.200 \\
\mathrm{j}-\mathrm{q}\end{array}$ & $\begin{array}{c}12.900 \\
\mathrm{k}-\mathrm{q}\end{array}$ & $\begin{array}{c}13.733 \\
\text { e-g }\end{array}$ & \multirow{3}{*}{$\begin{array}{c}14.788 \\
\text { bc }\end{array}$} & & & $\begin{array}{c}16.866 \\
\text { bc }\end{array}$ & $\begin{array}{c}14.800 \\
\text { de }\end{array}$ & $\begin{array}{c}12.700 \\
\text { fg }\end{array}$ \\
\hline & & 100 & $\begin{array}{c}17.200 \\
\text { b-h }\end{array}$ & $\begin{array}{c}15.100 \\
\text { g-n }\end{array}$ & $\begin{array}{c}12.100 \\
n-t\end{array}$ & $\begin{array}{c}14.800 \\
\mathrm{de}\end{array}$ & & & & & & \\
\hline & & 200 & 18.300 & 16.100 & 13.100 & 15.833 & & & & & & \\
\hline
\end{tabular}


$\underline{\text { Journal of Education and Science (ISSN 1812-125X), Vol: 29, No: 2, } 2020(260-278)}$

\begin{tabular}{|c|c|c|c|c|c|c|c|c|c|c|c|c|c|}
\hline & \multirow{4}{*}{ Sunflower } & \multirow[b]{2}{*}{0} & b-f & $d-j$ & $j-q$ & $\mathrm{~cd}$ & \multirow{4}{*}{$\begin{array}{c}15.548 \\
\mathrm{~b}\end{array}$} & & & & & \\
\hline & & & & $\begin{array}{c}15.200 \\
\text { g-n }\end{array}$ & $\begin{array}{c}13.900 \\
j-p\end{array}$ & $\begin{array}{c}10.800 \\
\mathrm{p}-\mathrm{t}\end{array}$ & $\begin{array}{c}13.300 \\
\text { e-h }\end{array}$ & & & & $\begin{array}{c}18.033 \\
\mathrm{~b}\end{array}$ & $\begin{array}{c}16.077 \\
\mathrm{~cd}\end{array}$ & $\begin{array}{c}12.533 \\
\text { fg }\end{array}$ \\
\hline & & & 100 & $\begin{array}{c}18.700 \\
\text { b-d }\end{array}$ & $\begin{array}{c}16.200 \\
\mathrm{~d}-\mathrm{j}\end{array}$ & $\begin{array}{c}12.300 \\
\mathrm{~m}-\mathrm{s}\end{array}$ & $\begin{array}{c}15.733 \\
\mathrm{~cd}\end{array}$ & & & & & & \\
\hline & & & 200 & $\begin{array}{c}20.200 \\
b\end{array}$ & $\begin{array}{c}18.133 \\
\text { b-g }\end{array}$ & $\begin{array}{c}14.500 \\
\text { h-n }\end{array}$ & $\begin{array}{c}17.611 \\
\text { ab }\end{array}$ & & & & & & \\
\hline & & & & & & & & Cultivar & Residues & Ascorbic & Residue & $\begin{array}{l}\times \text { Field C } \\
\text { Aserobi }\end{array}$ & pacity $\times$ \\
\hline $\begin{array}{r}\text { Culti } \\
\times\end{array}$ & & IP & & $\begin{array}{c}16.460 \\
b\end{array}$ & $\begin{array}{c}14.300 \\
\mathrm{c}\end{array}$ & $\begin{array}{c}11.281 \\
\mathrm{e}\end{array}$ & & $\begin{array}{c}14.014 \\
\mathrm{~b}\end{array}$ & & & & & \\
\hline $\begin{array}{r}\text { Fie } \\
\text { Capa } \\
\end{array}$ & & Shan & & $\begin{array}{c}18.596 \\
\mathrm{a} \\
\end{array}$ & $\begin{array}{c}15.951 \\
\mathrm{~b}\end{array}$ & $\begin{array}{c}13.166 \\
\mathrm{~d}\end{array}$ & & $\begin{array}{c}15.904 \\
\mathrm{a} \\
\end{array}$ & & & & & \\
\hline Resid & & Con & & $\begin{array}{c}19.344 \\
\mathrm{a} \\
\end{array}$ & $\begin{array}{c}15.838 \\
\mathrm{c}\end{array}$ & $\begin{array}{c}13.188 \\
\mathrm{e}\end{array}$ & & & $\begin{array}{c}16.124 \\
\mathrm{a}\end{array}$ & & & & \\
\hline $\begin{array}{r}\times \\
\mathrm{Fie}\end{array}$ & & Col & & $\begin{array}{c}15.916 \\
\mathrm{c}\end{array}$ & $\begin{array}{c}14.261 \\
\mathrm{~d}\end{array}$ & $\begin{array}{c}11.500 \\
\mathrm{f}\end{array}$ & & & $\begin{array}{c}13.892 \\
\mathrm{c}\end{array}$ & & & & \\
\hline Capa & & Sunflc & & $\begin{array}{c}17.327 \\
\mathrm{~b}\end{array}$ & $\begin{array}{c}15.277 \\
\mathrm{~cd}\end{array}$ & $\begin{array}{c}11.983 \\
\mathrm{f}\end{array}$ & & & $\begin{array}{c}14.860 \\
\mathrm{~b}\end{array}$ & & & & \\
\hline Asco & & 0 & & $\begin{array}{c}15.400 \\
\mathrm{~d}\end{array}$ & $\begin{array}{c}13.472 \\
\mathrm{e}\end{array}$ & $\begin{array}{c}10.983 \\
\mathrm{~g}\end{array}$ & & & & $\begin{array}{c}13.285 \\
\mathrm{c}\end{array}$ & & & \\
\hline $\begin{array}{r}\times \\
\mathrm{Fie}\end{array}$ & & 10 & & $\begin{array}{c}17.833 \\
\mathrm{~b} \\
\end{array}$ & $\begin{array}{c}15.283 \\
\mathrm{~d}\end{array}$ & $\begin{array}{c}12.188 \\
\mathrm{f}\end{array}$ & & & & $\begin{array}{c}15.101 \\
b\end{array}$ & & & \\
\hline Capa & & 20 & & $\begin{array}{c}19.355 \\
\mathrm{a}\end{array}$ & $\begin{array}{c}16.622 \\
\mathrm{c}\end{array}$ & $\begin{array}{c}13.500 \\
\mathrm{e}\end{array}$ & & & & $\begin{array}{c}16.492 \\
\mathrm{a} \\
\end{array}$ & & & \\
\hline & & 0 & & $\begin{array}{c}14.200 \\
\text { ef }\end{array}$ & $\begin{array}{c}12.344 \\
\mathrm{~g}\end{array}$ & $\begin{array}{c}9.500 \\
\mathrm{~h}\end{array}$ & & & & 0 & $\begin{array}{c}17.550 \\
\text { c-e }\end{array}$ & $\begin{array}{c}14.600 \\
\mathrm{f}-\mathrm{i}\end{array}$ & $\begin{array}{c}11.700 \\
\mathrm{k}-\mathrm{m}\end{array}$ \\
\hline ? & $\stackrel{\Omega}{a}$ & 10 & & $\begin{array}{c}17.066 \\
\text { b-d }\end{array}$ & $\begin{array}{c}14.766 \\
\mathrm{e}\end{array}$ & $\begin{array}{c}11.611 \\
\mathrm{~g}\end{array}$ & & & Control & 100 & $\begin{array}{c}19.150 \\
b c\end{array}$ & $\begin{array}{c}15.800 \\
\mathrm{e}-\mathrm{g}\end{array}$ & $\begin{array}{c}13.316 \\
\mathrm{i}-\mathrm{k}\end{array}$ \\
\hline 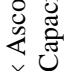 & & 20 & & $\begin{array}{c}18.122 \\
b c\end{array}$ & $\begin{array}{c}15.788 \\
\text { de }\end{array}$ & $\begin{array}{c}12.733 \\
\text { fg }\end{array}$ & & & & 200 & $\begin{array}{c}21.33 \\
\mathrm{a} \\
\end{array}$ & $\begin{array}{c}17.116 \\
\text { de }\end{array}$ & $\begin{array}{c}14.550 \\
\mathrm{f}-\mathrm{i}\end{array}$ \\
\hline 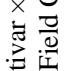 & & 0 & & $\begin{array}{c}16.600 \\
\mathrm{~cd}\end{array}$ & $\begin{array}{c}14.600 \\
\mathrm{e}\end{array}$ & $\begin{array}{c}12.466 \\
\mathrm{~g}\end{array}$ & & & & 0 & $\begin{array}{c}14.200 \\
\mathrm{~g}-\mathrm{i}\end{array}$ & $\begin{array}{c}12.700 \\
\mathrm{i}-1\end{array}$ & $\begin{array}{c}11.100 \\
\mathrm{im}\end{array}$ \\
\hline $\bar{\Xi} \bar{x}$ & 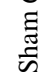 & 10 & & $\begin{array}{c}18.600 \\
\mathrm{~b}\end{array}$ & $\begin{array}{c}15.800 \\
\mathrm{de}\end{array}$ & $\begin{array}{c}12.766 \\
\mathrm{fg}\end{array}$ & & & Corn & 100 & $\begin{array}{c}16.350 \\
\text { d-f }\end{array}$ & $\begin{array}{c}14.400 \\
\mathrm{f}-\mathrm{i}\end{array}$ & $\begin{array}{c}11.150 \\
\mathrm{im}\end{array}$ \\
\hline & & 20 & & $\begin{array}{c}20.588 \\
\mathrm{a} \\
\end{array}$ & $\begin{array}{c}17.455 \\
\text { bc }\end{array}$ & $\begin{array}{c}14.266 \\
\text { ef }\end{array}$ & & & & 200 & $\begin{array}{c}17.200 \\
\mathrm{de}\end{array}$ & $\begin{array}{c}15.683 \\
\text { e-h }\end{array}$ & $\begin{array}{c}12.250 \\
\mathrm{j}-\mathrm{i}\end{array}$ \\
\hline & & & & & & & & & & 0 & $\begin{array}{c}14.450 \\
\text { f-i }\end{array}$ & $\begin{array}{c}13.116 \\
\text { i-1 }\end{array}$ & $\begin{array}{c}10.150 \\
\mathrm{~m}\end{array}$ \\
\hline $\begin{array}{r}\text { Fie } \\
\text { Capa }\end{array}$ & & & & $\begin{array}{c}17.529 \\
\mathrm{a}\end{array}$ & $\begin{array}{c}15.125 \\
\mathrm{~b}\end{array}$ & $\begin{array}{c}12.224 \\
\mathrm{c}\end{array}$ & & & Sunflower & 100 & $\begin{array}{c}18.000 \\
\text { b-d }\end{array}$ & $\begin{array}{c}15.650 \\
\text { e-h }\end{array}$ & $\begin{array}{c}12.100 \\
\mathrm{k}-\mathrm{m}\end{array}$ \\
\hline & & & & & & & & & & 200 & $\begin{array}{c}19.533 \\
\text { b }\end{array}$ & $\begin{array}{c}17.066 \\
\mathrm{de}\end{array}$ & $\begin{array}{c}13.700 \\
\text { h-k }\end{array}$ \\
\hline
\end{tabular}

decrease in relative water content of wheat compared to the control treatment. Lambers et al. (1998) [27] explained that inhibition is due to the release of allelopathic compounds from residues to soil and most of these compounds have the potential to dissolve in water and move through the root to the plant. The results also show that spraying plants with ascorbic acid has a positive effect, a stimulation was obtained at $200 \mathrm{ppm}$ concentration compared with $100 \mathrm{ppm}$ and zero, the stimulation rate was $(13.9,22.6 \%)$ respectively. The results are consistent with Al-Obaidy (2015) [15] the stimulation of wheat seeds with ascorbic acid caused a significant increase in this trait, superior to control treatment.

The effect of ascorbic acid is attributed to the positive effect on the relative water content, it was positively correlated with phenols and proline content. The indicators of membranes stability are also improved when the seeds are treated with ascorbic acid [28]. The results of the interaction between the field capacity and the ascorbic indicate that the field capacity $85 \%$ exceeded at 200 ppm concentration.

With regard to triangular interaction (cultivar $\times$ ascorbic $\times$ residues) the wheat cultivar sham 6 at a concentration of $200 \mathrm{ppm}$ was superior in treatment without residues. The interaction (cultivar $\times$ ascorbic $\times$ residues $\times$ field capacity) sham 6 was superior at field capacity $85 \%$ in corn residues ascorbic acid and at concentration $200 \mathrm{ppm}$. 


\section{Chlorophyll and Carotenoid Content}

Results of tables $(4,5)$ indicated the average of chlorophyll and carotenoid content was significantly decreased by water deficit and the reduction was significantly decreased with the increase of water deficit stress. The maximum decrease recorded at $35 \%$ of field capacity (17.0, $34.3 \%$ ) compared to the treatment $85 \%$ respectively.

Drought is the most environmental factors that have limited plant growth. The low content of plant pigment is attributed to the wet soils that are not strongly held by water whereas dry soils are held or constrained, so we need force to extract water from the soil also water may be led to stomata closure, which reduce $\mathrm{CO}_{2}$ availability in leaves and inhibits carbon fixation leading to the reduction plant pigment $[29,30]$.

These results are in agreement with those obtained by Movani (2011) [31] indicated the lower chlorophyll content in wheat due to the water stress compared to control treatment.

Saker (2010) [32] explained that demolition enzymes chlorophylls activated under stress conditions and distort the size and shape of chloroplasts and reduce of carotenoid, which has a significant role in protecting chlorophyll from demolition under water stress. The addition of the two crop residues (corn, sunflower) caused a reduction in values of total chlorophyll content compared with control, while they showed no significant differences in carotenoid content. The existence of residues plant leads to the increasing the of concentration of allelopathic compounds, which may be related to decline the level of enzymes that are essential for the construction of chlorophyll structure [24]. Rice (1984) [4] proposed that some allelopathic compounds may inhibit synthesis porphyrin which is the basic molecule in chlorophyll. This was confirmed by Ibrahim et al. (2013) [33] a study that shows that the allelopathic effects of extract prepared from genetically modified corn significantly decreased chlorophyll and carotenoid content of wheat.

Results of the table indicated that spraying plants with ascorbic acid caused a significant increase in chlorophyll compared to the control while no significant increase in carotenoid. This may be due to the physiological role of ascorbic acid in

Table (3) Effect of corn, sunflower residues and field capacity in relative water content (\%) of wheat treated with ascorbic acid

\begin{tabular}{|c|c|c|c|c|c|c|c|c|c|c|c|c|}
\hline \multirow[b]{2}{*}{ Cultivar } & \multirow[b]{2}{*}{ Residues } & \multirow{2}{*}{$\begin{array}{l}\text { Ascorbic } \\
\text { acid }\end{array}$} & \multicolumn{3}{|c|}{ Field Capacity } & \multirow{2}{*}{$\begin{array}{l}\text { Cultivar } \\
\times \\
\text { Residues } \\
\times \\
\text { Ascorbic }\end{array}$} & \multirow{2}{*}{$\begin{array}{l}\text { Cultivar } \\
\times \\
\text { Residues }\end{array}$} & \multirow{2}{*}{$\begin{array}{l}\text { Cultivar } \\
\times \\
\text { Ascorbic }\end{array}$} & \multirow{2}{*}{$\begin{array}{l}\text { Residues } \\
\times \\
\text { Ascorbic }\end{array}$} & \multicolumn{3}{|c|}{$\begin{array}{l}\text { Cultivar } \times \text { Residues } \times \text { Field } \\
\text { Capacity }\end{array}$} \\
\hline & & & 85 & 60 & 35 & & & & & 85 & 60 & 35 \\
\hline \multirow{9}{*}{ IPA 99} & \multirow{3}{*}{ Control } & 0 & $\begin{array}{l}72.700 \\
\text { f-h }\end{array}$ & $\begin{array}{l}59.900 \\
\mathrm{~m}-\mathrm{o}\end{array}$ & $\begin{array}{l}51.100 \\
\text { s-u }\end{array}$ & $\begin{array}{l}61.233 \\
\mathrm{f}\end{array}$ & \multirow{3}{*}{$\begin{array}{l}66.544 \\
b\end{array}$} & $\begin{array}{l}55.296 \\
e^{-29}\end{array}$ & $\begin{array}{l}63.638 \\
d\end{array}$ & $\begin{array}{l}78.166 \\
b\end{array}$ & $\begin{array}{l}64.700 \\
e^{-6}\end{array}$ & $\begin{array}{l}56.766 \\
\text { hi }\end{array}$ \\
\hline & & 100 & $\begin{array}{l}73.400 \\
\text { fg }\end{array}$ & $\begin{array}{l}62.100 \\
\text { im }\end{array}$ & $\begin{array}{l}58.100 \\
n-q\end{array}$ & $\begin{array}{l}64.533 \\
\text { de }\end{array}$ & & $\begin{array}{l}57.185 \\
d\end{array}$ & $\begin{array}{l}67.783 \\
\mathrm{c}\end{array}$ & & & \\
\hline & & 200 & $\begin{array}{l}88.400 \\
b\end{array}$ & $\begin{array}{l}72.100 \\
g-i\end{array}$ & $\begin{array}{l}61.000 \\
\text { i-n }\end{array}$ & $\begin{array}{l}73.866 \\
\mathrm{a}\end{array}$ & & \begin{tabular}{|l|}
70.444 \\
$\mathrm{a}$
\end{tabular} & $\begin{array}{l}73.150 \\
\mathrm{a}\end{array}$ & & & \\
\hline & \multirow{3}{*}{ Corn } & 0 & $\begin{array}{l}52.900 \\
\mathrm{r}-\mathrm{t}\end{array}$ & $\begin{array}{l}51.167 \\
\text { s-u }\end{array}$ & $36.500 \mathrm{z}$ & $\begin{array}{l}46.855 \\
j\end{array}$ & \multirow{3}{*}{$\begin{array}{l}54.703 \\
\mathrm{f}\end{array}$} & & $\begin{array}{l}52.055 \\
\mathrm{~g}\end{array}$ & $\begin{array}{l}59.888 \\
\mathrm{~g}\end{array}$ & $\begin{array}{l}58.188 \\
\text { gh }\end{array}$ & $\begin{array}{l}46.033 \\
\mathrm{k}\end{array}$ \\
\hline & & 100 & \begin{tabular}{|l|}
55.767 \\
p-r \\
\end{tabular} & \begin{tabular}{|l|}
54.400 \\
q-s \\
\end{tabular} & \begin{tabular}{|l|}
44.500 \\
$w-y$ \\
\end{tabular} & $\begin{array}{l}51.555 \\
\mathrm{i} \\
\end{array}$ & & & \begin{tabular}{|l|}
58.866 \\
$\mathrm{e}$ \\
\end{tabular} & & & \\
\hline & & 200 & $\begin{array}{l}71.000 \\
\mathrm{~g}-\mathrm{i}\end{array}$ & $\begin{array}{l}69.000 \\
h-j\end{array}$ & $\begin{array}{l}57.100 \\
0-q\end{array}$ & $\begin{array}{l}\text { 65.700 } \\
\text { de }\end{array}$ & & & $\begin{array}{l}68.916 \\
b c\end{array}$ & & & \\
\hline & \multirow{3}{*}{ Sunflower } & 0 & $\begin{array}{l}60.800 \\
\mathrm{i}-\mathrm{o}\end{array}$ & $\begin{array}{l}59.600 \\
\text { m-o }\end{array}$ & $\begin{array}{l}53.000 \\
\mathrm{r}-\mathrm{t}\end{array}$ & $\begin{array}{l}57.800 \\
\mathrm{~g}\end{array}$ & \multirow{3}{*}{$\begin{array}{l}61.677 \\
\mathrm{e}\end{array}$} & & $\begin{array}{l}57.388 \\
\mathrm{f} \\
\end{array}$ & $\begin{array}{l}74.000 \\
\mathrm{c}\end{array}$ & $\begin{array}{l}62.600 \\
\mathrm{f}\end{array}$ & $\begin{array}{l}48.433 \\
j\end{array}$ \\
\hline & & 100 & $\begin{array}{l}64.100 \\
\mathrm{kl}\end{array}$ & $\begin{array}{l}61.100 \\
\text { i-n }\end{array}$ & $\begin{array}{l}41.200 \\
y\end{array}$ & $\begin{array}{l}55.466 \\
h\end{array}$ & & & \begin{tabular}{|l|}
59.600 \\
$\mathrm{e}$
\end{tabular} & & & \\
\hline & & 200 & $\begin{array}{l}97.100 \\
\mathrm{a}\end{array}$ & $\begin{array}{l}67.100 \\
\mathrm{jk}\end{array}$ & $\begin{array}{l}51.100 \\
\text { s-u }\end{array}$ & $\begin{array}{l}71.766 \\
b\end{array}$ & & & 70.166 & & & \\
\hline \multirow{3}{*}{ Sham 6} & \multirow{3}{*}{ Control } & 0 & $\begin{array}{l}75.700 \\
\text { ef }\end{array}$ & $\begin{array}{l}71.233 \\
\mathrm{~g}-\mathrm{i}\end{array}$ & \begin{tabular}{|l|}
51.200 \\
s-u
\end{tabular} & $\begin{array}{l}66.044 \\
d\end{array}$ & \multirow{3}{*}{$\begin{array}{l}69.837 \\
\mathrm{a}\end{array}$} & $\begin{array}{l}60.092 \\
\mathrm{c}\end{array}$ & & $\begin{array}{l}78.500 \\
b\end{array}$ & $e^{75.144}$ & $\begin{array}{l}55.866 \\
\mathrm{i}\end{array}$ \\
\hline & & 100 & $\begin{array}{l}79.700 \\
\text { d }\end{array}$ & $\begin{array}{l}76.100 \\
\text { ef }\end{array}$ & $\begin{array}{l}57.300 \\
n-q\end{array}$ & $\begin{array}{l}71.033 \\
\mathrm{~b}\end{array}$ & & $\begin{array}{l}66.981 \\
b\end{array}$ & & & & \\
\hline & & 200 & $\begin{array}{l}80.100 \\
d\end{array}$ & $\begin{array}{l}78.100 \\
\mathrm{de}\end{array}$ & \begin{tabular}{|l|}
59.100 \\
$m-p$
\end{tabular} & $\begin{array}{l}72.433 \\
\mathrm{ab}\end{array}$ & & \begin{tabular}{|l|}
71.044 \\
$\mathrm{a}$
\end{tabular} & & & & \\
\hline
\end{tabular}


$\underline{\text { Journal of Education and Science (ISSN 1812-125X), Vol: 29, No: 2, } 2020(260-278)}$

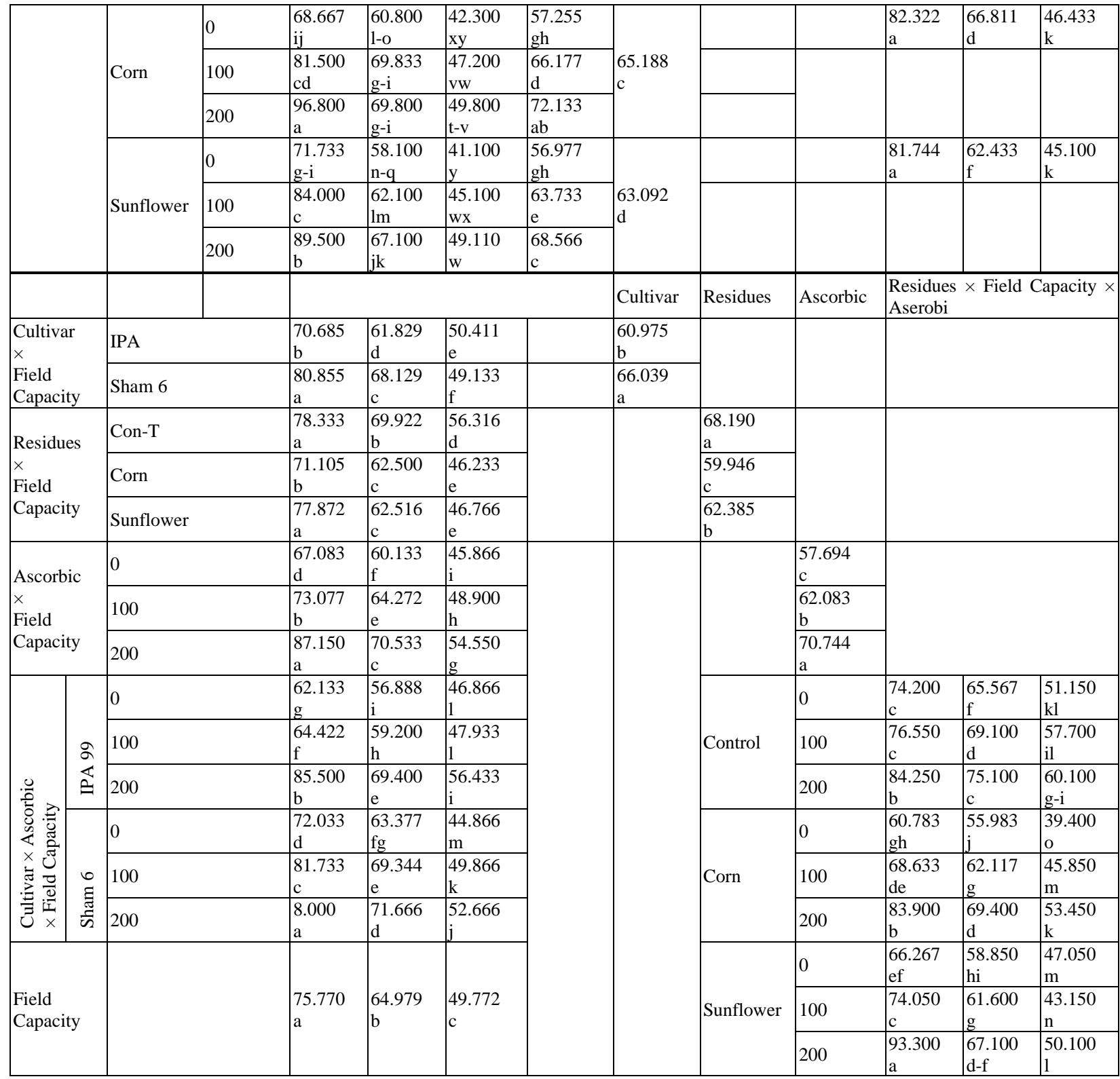

Table (4) Effect of corn, sunflower residues and field capacity in total chlorophyll (mg/g) of wheat treated with ascorbic acid

\begin{tabular}{|c|c|c|c|c|c|c|c|c|c|c|c|c|}
\hline \multirow[b]{2}{*}{ Cultivar } & \multirow[b]{2}{*}{ Residues } & \multirow{2}{*}{$\begin{array}{l}\text { Ascorbic } \\
\text { acid }\end{array}$} & \multicolumn{3}{|c|}{ Field Capacity } & \multirow{2}{*}{$\begin{array}{l}\text { Cultivar } \\
\times \\
\text { Residues } \\
\times \\
\text { Ascorbic }\end{array}$} & \multirow{2}{*}{$\begin{array}{l}\text { Cultivar } \\
\times \\
\text { Residues }\end{array}$} & \multirow{2}{*}{$\begin{array}{l}\text { Cultivar } \\
\times \\
\text { Ascorbic }\end{array}$} & \multirow{2}{*}{$\begin{array}{l}\text { Residues } \\
\times \\
\text { Ascorbic }\end{array}$} & \multicolumn{3}{|c|}{$\begin{array}{l}\text { Cultivar } \times \text { Residues } \times \text { Field } \\
\text { Capacity }\end{array}$} \\
\hline & & & 85 & 60 & 35 & & & & & 85 & 60 & 35 \\
\hline \multirow{8}{*}{ IPA 99} & \multirow{3}{*}{ Control } & 0 & $\begin{array}{l}2.810 \\
d-j\end{array}$ & $\begin{array}{l}2.770 \\
\mathrm{~d}-\mathrm{k}\end{array}$ & $\begin{array}{l}2.320 \\
k-n\end{array}$ & $\begin{array}{l}2.633 \\
e-h\end{array}$ & \multirow{3}{*}{$\begin{array}{l}2.777 \\
c\end{array}$} & $\begin{array}{l}2.538 \\
D\end{array}$ & $\begin{array}{l}2.768 \\
\mathrm{~cd}\end{array}$ & $\begin{array}{l}3.038 \\
\mathrm{bc}\end{array}$ & $\begin{array}{l}2.830 \\
c-e\end{array}$ & $\begin{array}{l}2.463 \\
\mathrm{fg}\end{array}$ \\
\hline & & 100 & $\begin{array}{l}2.996 \\
\mathrm{c}-\mathrm{g}\end{array}$ & $\begin{array}{l}2.810 \\
d-j\end{array}$ & $\begin{array}{l}2.450 \\
\mathrm{j}-\mathrm{n}\end{array}$ & $\begin{array}{l}2.752 \\
\mathrm{c}-\mathrm{g}\end{array}$ & & $\begin{array}{l}2.666 \\
\mathrm{C}\end{array}$ & $\begin{array}{l}2.846 \\
\mathrm{bc}\end{array}$ & & & \\
\hline & & 200 & $\begin{array}{l}3.310 \\
\mathrm{a}-\mathrm{c}\end{array}$ & $\begin{array}{l}2.910 \\
c-j\end{array}$ & $\begin{array}{l}2.620 \\
\mathrm{~g}-\mathrm{m}\end{array}$ & $\begin{array}{l}2.946 \\
\mathrm{bc}\end{array}$ & & $\begin{array}{l}2.787 \\
\mathrm{Bc}\end{array}$ & $\begin{array}{l}3.211 \\
\mathrm{a}\end{array}$ & & & \\
\hline & \multirow{3}{*}{ Corn } & 0 & $\begin{array}{l}2.710 \\
\mathrm{~d}-\mathrm{k}\end{array}$ & $\begin{array}{l}2.620 \\
\text { g-m }\end{array}$ & $\begin{array}{l}2.110 \\
\mathrm{n}\end{array}$ & $\begin{array}{l}2.480 \\
\mathrm{~h}\end{array}$ & \multirow{3}{*}{$\begin{array}{l}2.571 \\
d\end{array}$} & & $\begin{array}{l}2.628 \\
\mathrm{~d}\end{array}$ & $\begin{array}{l}2.803 \\
\mathrm{c}-\mathrm{e}\end{array}$ & $\begin{array}{l}2.711 \\
\mathrm{de}\end{array}$ & $\begin{array}{l}2.201 \\
\mathrm{~h}\end{array}$ \\
\hline & & 100 & $\begin{array}{l}2.790 \\
\mathrm{~d}-\mathrm{j} \\
\end{array}$ & $\begin{array}{l}2.703 \\
\mathrm{~d}-\mathrm{k}\end{array}$ & $\begin{array}{l}2.213 \\
\mathrm{mn}\end{array}$ & $\begin{array}{l}2.568 \\
\text { f-h } \\
\end{array}$ & & & \begin{tabular}{|l|}
2.699 \\
$\mathrm{~cd}$
\end{tabular} & & & \\
\hline & & 200 & $\begin{array}{l}2.910 \\
c-j\end{array}$ & $\begin{array}{l}2.810 \\
\mathrm{~d}-\mathrm{j}\end{array}$ & $\begin{array}{l}2.280 \\
1-n \\
\end{array}$ & $\begin{array}{l}2.666 \\
e-g\end{array}$ & & & $\begin{array}{l}2.827 \\
\mathrm{bc}\end{array}$ & & & \\
\hline & \multirow[t]{2}{*}{ Sunflower } & 0 & $\begin{array}{l}2.610 \\
\mathrm{~g}-\mathrm{m}\end{array}$ & $\begin{array}{l}2.590 \\
\text { g-m }\end{array}$ & $\begin{array}{l}2.310 \\
k-n\end{array}$ & $\begin{array}{l}2.503 \\
\mathrm{gh}\end{array}$ & \multirow{2}{*}{$\begin{array}{l}2.642 \\
d\end{array}$} & & $\begin{array}{l}2.625 \\
\mathrm{~d}\end{array}$ & $\begin{array}{l}2.844 \\
\mathrm{~cd}\end{array}$ & $\begin{array}{l}2.706 \\
\mathrm{de}\end{array}$ & $\begin{array}{l}2.377 \\
\mathrm{gh}\end{array}$ \\
\hline & & 100 & 2.820 & 2.710 & 2.503 & 2.677 & & & 2.792 & & & \\
\hline
\end{tabular}


$\underline{\text { Journal of Education and Science (ISSN 1812-125X), Vol: 29, No: 2, } 2020(260-278)}$

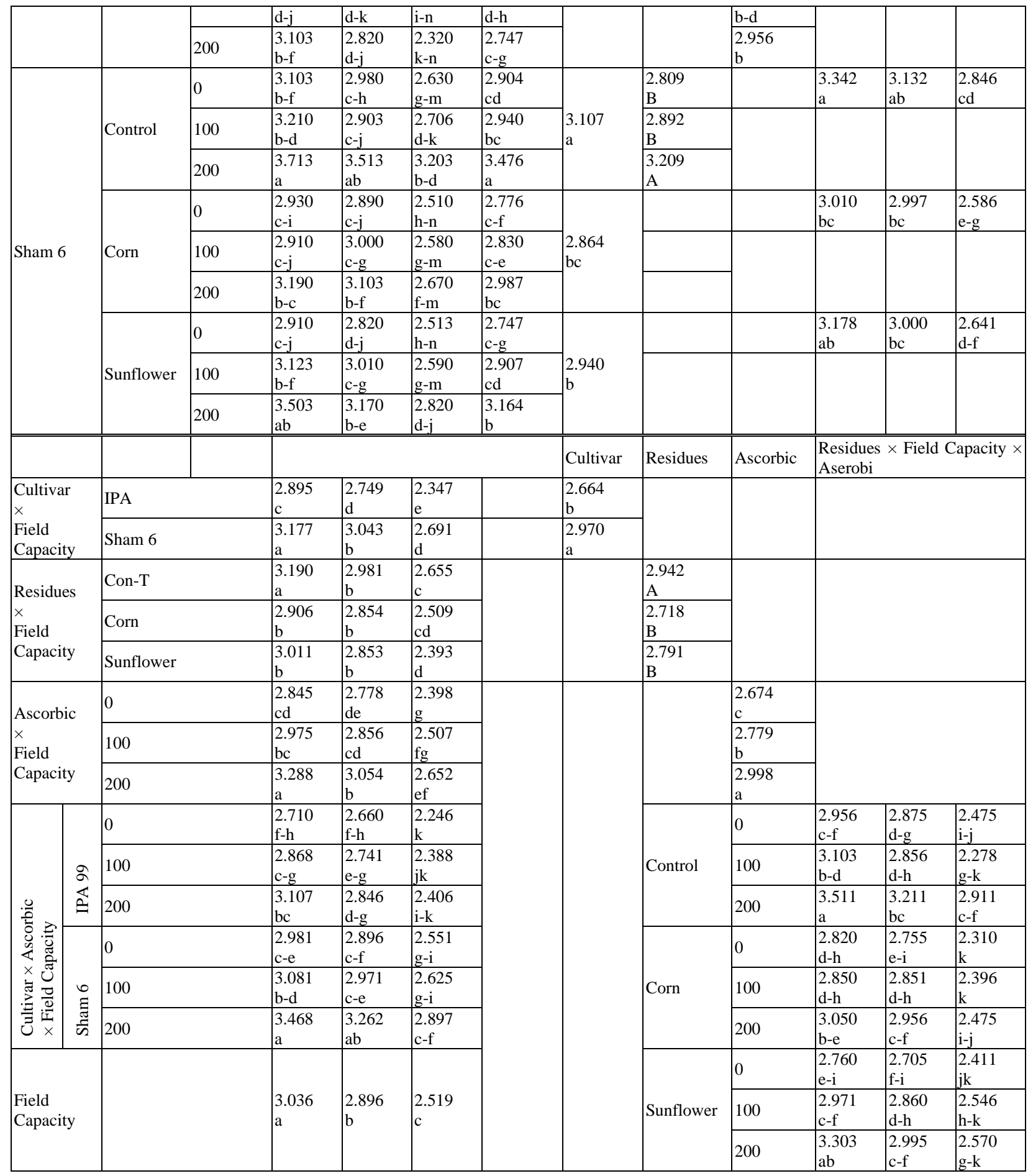

Table (5) Effect of corn, sunflower residues and field capacity in carotenoid (mg/g) of wheat treated with ascorbic acid

\begin{tabular}{|c|c|c|c|c|c|c|c|c|c|c|c|c|}
\hline \multirow[b]{2}{*}{ Cultivar } & \multirow[b]{2}{*}{ Residues } & \multirow{2}{*}{$\begin{array}{l}\text { Ascorbic } \\
\text { acid }\end{array}$} & \multicolumn{3}{|c|}{ Field Capacity } & \multirow{2}{*}{\begin{tabular}{|l|} 
Cultivar \\
$\times$ \\
Residues \\
$\times$ \\
Ascorbic \\
\end{tabular}} & \multirow{2}{*}{$\begin{array}{l}\text { Cultivar } \\
\times \\
\text { Residues }\end{array}$} & \multirow{2}{*}{$\begin{array}{l}\text { Cultivar } \\
\times \\
\text { Ascorbic }\end{array}$} & \multirow{2}{*}{$\begin{array}{l}\text { Residues } \\
\times \\
\text { Ascorbic }\end{array}$} & \multicolumn{3}{|c|}{$\begin{array}{l}\text { Cultivar } \times \text { Residues } \times \text { Field } \\
\text { Capacity }\end{array}$} \\
\hline & & & 85 & 60 & 35 & & & & & 85 & 60 & 35 \\
\hline \multirow{2}{*}{ IPA 99} & \multirow{2}{*}{ Control } & 0 & $\begin{array}{l}0.891 \\
\text { a-g }\end{array}$ & $\begin{array}{l}0.882 \\
\text { a-h }\end{array}$ & $\begin{array}{l}0.611 \\
\mathrm{~d}-\mathrm{k}\end{array}$ & $\begin{array}{l}0.794 \\
\mathrm{a}\end{array}$ & \multirow{2}{*}{$\begin{array}{l}0.815 \\
\mathrm{a}\end{array}$} & $\begin{array}{l}0.766 \\
\mathrm{~A}\end{array}$ & $\begin{array}{l}0.797 \\
\mathrm{a}\end{array}$ & $\begin{array}{l}0.926 \\
\mathrm{a}\end{array}$ & $\begin{array}{l}0.896 \\
\mathrm{a}\end{array}$ & $\begin{array}{l}0.623 \\
\mathrm{~b}\end{array}$ \\
\hline & & 100 & $\begin{array}{l}0.921 \\
a-e\end{array}$ & $\begin{array}{l}0.911 \\
\text { a-f }\end{array}$ & $\begin{array}{l}0.632 \\
\mathrm{c}-\mathrm{k}\end{array}$ & $\begin{array}{l}0.821 \\
\mathrm{a}\end{array}$ & & $\begin{array}{l}0.790 \\
\mathrm{~A}\end{array}$ & $\begin{array}{l}0.776 \\
\mathrm{a}\end{array}$ & & & \\
\hline
\end{tabular}


$\underline{\text { Journal of Education and Science (ISSN 1812-125X), Vol: 29, No: 2, } 2020 \text { (260-278) }}$

\begin{tabular}{|c|c|c|c|c|c|c|c|c|c|c|c|c|c|}
\hline & & & 200 & $\begin{array}{l}0.966 \\
\mathrm{ab}\end{array}$ & $\begin{array}{l}0.897 \\
\mathrm{a}-\mathrm{g}\end{array}$ & $\begin{array}{l}0.627 \\
\mathrm{~d}-\mathrm{k}\end{array}$ & $\begin{array}{l}0.830 \\
\mathrm{a} \\
\end{array}$ & & $\begin{array}{l}0.817 \\
\mathrm{~A} \\
\end{array}$ & $\begin{array}{l}0.839 \\
\mathrm{a} \\
\end{array}$ & & & \\
\hline & & & 0 & $\begin{array}{l}0.854 \\
a-j\end{array}$ & $\begin{array}{l}0.872 \\
\text { a-h } \\
\end{array}$ & $\begin{array}{l}0.510 \\
\mathrm{k}\end{array}$ & $\begin{array}{l}0.745 \\
\mathrm{a} \\
\end{array}$ & & & \begin{tabular}{|l|}
0.775 \\
$\mathrm{a}$ \\
\end{tabular} & $\begin{array}{l}0.896 \\
\mathrm{a}\end{array}$ & $\begin{array}{l}0.876 \\
\mathrm{a}\end{array}$ & $\begin{array}{l}0.554 \\
\mathrm{~b}\end{array}$ \\
\hline & & Corn & 100 & $\begin{array}{l}0.890 \\
\mathrm{a}-\mathrm{g}\end{array}$ & $\begin{array}{l}0.872 \\
\text { a-h }\end{array}$ & $\begin{array}{l}0.561 \\
\text { i-k }\end{array}$ & $\begin{array}{l}0.774 \\
\mathrm{a}\end{array}$ & $\begin{array}{l}0.775 \\
a\end{array}$ & & \begin{tabular}{|l}
0.788 \\
$\mathrm{a}$
\end{tabular} & & & \\
\hline & & & 200 & $\begin{array}{l}0.944 \\
a-c\end{array}$ & $\begin{array}{l}0.885 \\
a-g\end{array}$ & $\begin{array}{l}0.591 \\
\mathrm{~g}-\mathrm{k}\end{array}$ & $\begin{array}{l}0.806 \\
\mathrm{a}\end{array}$ & & & $\begin{array}{l}0.831 \\
\mathrm{a}\end{array}$ & & & \\
\hline & & & 0 & $\begin{array}{l}0.891 \\
\mathrm{a}-\mathrm{g}\end{array}$ & $\begin{array}{l}0.873 \\
\text { a-h }\end{array}$ & $\begin{array}{l}0.511 \\
\mathrm{k}\end{array}$ & $\begin{array}{l}0.758 \\
\mathrm{a}\end{array}$ & & & $\begin{array}{l}0.764 \\
\mathrm{a}\end{array}$ & $\begin{array}{l}0.917 \\
\mathrm{a}\end{array}$ & $\begin{array}{l}0.875 \\
\mathrm{a}\end{array}$ & $\begin{array}{l}0.557 \\
\mathrm{~b}\end{array}$ \\
\hline & & Sunflower & 100 & $\begin{array}{l}0.630 \\
\mathrm{c}-\mathrm{k}\end{array}$ & $\begin{array}{l}0.867 \\
\mathrm{a}-\mathrm{i}\end{array}$ & $\begin{array}{l}0.570 \\
h-k\end{array}$ & $\begin{array}{l}0.776 \\
\mathrm{a}\end{array}$ & $\begin{array}{l}0.783 \\
a\end{array}$ & & $\begin{array}{l}0.790 \\
\mathrm{a}\end{array}$ & & & \\
\hline & & & 200 & $\begin{array}{l}0.972 \\
\mathrm{a} \\
\end{array}$ & $\begin{array}{l}0.887 \\
\text { a-g } \\
\end{array}$ & $\begin{array}{l}0.589 \\
\mathrm{~g}-\mathrm{k}\end{array}$ & $\begin{array}{l}0.816 \\
\mathrm{a}\end{array}$ & & & \begin{tabular}{|l|}
0.821 \\
$\mathrm{a}$ \\
\end{tabular} & & & \\
\hline & & & 0 & \begin{tabular}{|l}
0.894 \\
a-g \\
\end{tabular} & \begin{tabular}{|l|}
0.893 \\
a-g \\
\end{tabular} & $\begin{array}{l}0.612 \\
\mathrm{~d}-\mathrm{k}\end{array}$ & \begin{tabular}{|l}
0.800 \\
$\mathrm{a}$ \\
\end{tabular} & & $\begin{array}{l}0.778 \\
\mathrm{~A}\end{array}$ & & \begin{tabular}{|l}
0.843 \\
$\mathrm{a}$ \\
\end{tabular} & $\begin{array}{l}0.883 \\
\mathrm{a} \\
\end{array}$ & $\begin{array}{l}0.653 \\
\mathrm{~b}\end{array}$ \\
\hline & & Control & 100 & \begin{tabular}{|l}
0.890 \\
$\mathrm{a}-\mathrm{g}$ \\
\end{tabular} & \begin{tabular}{|l}
0.871 \\
a-h \\
\end{tabular} & \begin{tabular}{|l|}
0.694 \\
$\mathrm{a}-\mathrm{k}$
\end{tabular} & \begin{tabular}{|l}
0.731 \\
$\mathrm{a}$ \\
\end{tabular} & $\begin{array}{l}0.793 \\
\mathrm{a}\end{array}$ & $\begin{array}{l}0.779 \\
\mathrm{~A} \\
\end{array}$ & & & & \\
\hline & & & 200 & $\begin{array}{l}1.006 \\
\mathrm{a} \\
\end{array}$ & \begin{tabular}{|l}
0.887 \\
$\mathrm{a}-\mathrm{g}$ \\
\end{tabular} & $\begin{array}{l}0.651 \\
\mathrm{~b}-\mathrm{k}\end{array}$ & \begin{tabular}{|l}
0.848 \\
$\mathrm{a}$ \\
\end{tabular} & & \begin{tabular}{|l|}
0.843 \\
$\mathrm{~A}$ \\
\end{tabular} & & & & \\
\hline & & & 0 & $\begin{array}{l}0.873 \\
\text { a-h }\end{array}$ & $\begin{array}{l}0.872 \\
\text { a-h }\end{array}$ & $\begin{array}{l}0.551 \\
\mathrm{jk}\end{array}$ & $\begin{array}{l}0.765 \\
\mathrm{a}\end{array}$ & & & & $\begin{array}{l}0.932 \\
\mathrm{a}\end{array}$ & $\begin{array}{l}0.887 \\
\mathrm{a}\end{array}$ & $\begin{array}{l}0.613 \\
\mathrm{~b}\end{array}$ \\
\hline Sham & & Corn & 100 & $\begin{array}{l}0.936 \\
\text { a-d }\end{array}$ & $\begin{array}{l}0.879 \\
\text { a-h }\end{array}$ & $\begin{array}{l}0.591 \\
\mathrm{~g}-\mathrm{k}\end{array}$ & $\begin{array}{l}0.802 \\
\mathrm{a} \\
\end{array}$ & $\begin{array}{l}0.807 \\
\mathrm{a}\end{array}$ & & & & & \\
\hline & & & 200 & $\begin{array}{l}0.961 \\
\mathrm{ab}\end{array}$ & $\begin{array}{l}0.910 \\
\text { a-f }\end{array}$ & $\begin{array}{l}0.698 \\
\mathrm{a}-\mathrm{k}\end{array}$ & $\begin{array}{l}0.856 \\
\mathrm{a}\end{array}$ & & & & & & \\
\hline & & & 0 & $\begin{array}{l}0.896 \\
a-g\end{array}$ & $\begin{array}{l}0.882 \\
\text { a-h } \\
\end{array}$ & $\begin{array}{l}0.533 \\
\mathrm{k} \\
\end{array}$ & $\begin{array}{l}0.770 \\
\mathrm{a}\end{array}$ & & & & $\begin{array}{l}0.932 \\
\mathrm{a}\end{array}$ & $\begin{array}{l}0.894 \\
\mathrm{a}\end{array}$ & $\begin{array}{l}0.575 \\
b\end{array}$ \\
\hline & & Sunflower & 100 & $\begin{array}{l}0.913 \\
\text { a-f }\end{array}$ & \begin{tabular}{|l}
0.912 \\
a-f
\end{tabular} & $\begin{array}{l}0.591 \\
\text { g-k }\end{array}$ & $\begin{array}{l}0.805 \\
\mathrm{a}\end{array}$ & $\begin{array}{l}0.800 \\
\mathrm{a}\end{array}$ & & & & & \\
\hline & & & 200 & $\begin{array}{l}0.988 \\
\mathrm{a} \\
\end{array}$ & $\begin{array}{l}0.890 \\
\mathrm{a}-\mathrm{g}\end{array}$ & $\begin{array}{l}0.601 \\
\mathrm{f}-\mathrm{k} \\
\end{array}$ & $\begin{array}{l}0.826 \\
\mathrm{a} \\
\end{array}$ & & & & & & \\
\hline & & & & & & & & Cultivar & Residues & Ascorbic & $\begin{array}{l}\text { Residues } \\
\text { Aserobi }\end{array}$ & $\times$ Fielc & Tapacity $\times$ \\
\hline $\begin{array}{l}\text { Cultiva } \\
\times\end{array}$ & & IPA & & $\begin{array}{l}0.913 \\
\mathrm{a} \\
\end{array}$ & $\begin{array}{l}0.882 \\
\mathrm{a} \\
\end{array}$ & $\begin{array}{l}0.578 \\
\mathrm{~b}\end{array}$ & & $\begin{array}{l}0.791 \\
\mathrm{a}\end{array}$ & & & & & \\
\hline $\begin{array}{l}\text { Field } \\
\text { Capaci }\end{array}$ & & Sham 6 & & $\begin{array}{l}0.899 \\
\mathrm{a} \\
\end{array}$ & \begin{tabular}{|l}
0.888 \\
$\mathrm{a}$ \\
\end{tabular} & \begin{tabular}{|l}
0.613 \\
$\mathrm{~b}$ \\
\end{tabular} & & $\begin{array}{l}0.800 \\
\mathrm{a} \\
\end{array}$ & & & & & \\
\hline Residu & & Con-T & & $\begin{array}{l}0.884 \\
\mathrm{a} \\
\end{array}$ & $\begin{array}{l}0.890 \\
\mathrm{a} \\
\end{array}$ & $\begin{array}{l}\begin{array}{l}0.638 \\
\mathrm{~b}\end{array} \\
\end{array}$ & & & $\begin{array}{l}0.804 \\
\mathrm{~A} \\
\end{array}$ & & & & \\
\hline $\begin{array}{l}x \\
\text { Field }\end{array}$ & & Corn & & $\begin{array}{l}0.909 \\
\mathrm{a} \\
\end{array}$ & $\begin{array}{l}0.881 \\
\mathrm{a} \\
\end{array}$ & $\begin{array}{l}0.583 \\
\mathrm{~b}\end{array}$ & & & \begin{tabular}{|l}
0.791 \\
$\mathrm{~A}$ \\
\end{tabular} & & & & \\
\hline Capaci & & Sunflower & & $\begin{array}{l}0.925 \\
\mathrm{a} \\
\end{array}$ & $\begin{array}{l}0.885 \\
\mathrm{a} \\
\end{array}$ & $\begin{array}{l}0.566 \\
\mathrm{~b}\end{array}$ & & & $\begin{array}{l}0.792 \\
\mathrm{~A}\end{array}$ & & & & \\
\hline Ascorb & & 0 & & $\begin{array}{l}0.883 \\
\mathrm{a} \\
\end{array}$ & $\begin{array}{l}0.879 \\
\mathrm{a} \\
\end{array}$ & $\begin{array}{l}0.554 \\
\mathrm{~b}\end{array}$ & & & & $\begin{array}{l}0.772 \\
\mathrm{a}\end{array}$ & & & \\
\hline $\begin{array}{l}\times \\
\text { Field }\end{array}$ & & 100 & & $\begin{array}{l}0.863 \\
\mathrm{a}\end{array}$ & $\begin{array}{l}0.885 \\
\mathrm{a} \\
\end{array}$ & $\begin{array}{l}0.606 \\
b\end{array}$ & & & & $\begin{array}{l}0.785 \\
\mathrm{a} \\
\end{array}$ & & & \\
\hline Capaci & & 200 & & $\begin{array}{l}0.972 \\
\mathrm{a} \\
\end{array}$ & $\begin{array}{l}0.892 \\
\mathrm{a} \\
\end{array}$ & $\begin{array}{l}0.626 \\
\mathrm{~b}\end{array}$ & & & & $\begin{array}{l}0.830 \\
\mathrm{a} \\
\end{array}$ & & & \\
\hline & & 0 & & \begin{tabular}{|l}
0.878 \\
$\mathrm{a}$ \\
\end{tabular} & $\begin{array}{l}0.875 \\
\mathrm{a} \\
\end{array}$ & \begin{tabular}{|l}
0.544 \\
$\mathrm{~b}$ \\
\end{tabular} & & & & 0 & $\begin{array}{l}0.892 \\
\mathrm{a} \\
\end{array}$ & $\begin{array}{l}0.887 \\
\mathrm{a} \\
\end{array}$ & $\begin{array}{l}0.612 \\
\mathrm{bc}\end{array}$ \\
\hline & ลे & 100 & & \begin{tabular}{|l}
0.900 \\
$\mathrm{a}$ \\
\end{tabular} & \begin{tabular}{|l}
0.883 \\
$\mathrm{a}$ \\
\end{tabular} & \begin{tabular}{|l}
$\begin{array}{l}0.588 \\
\mathrm{~b}\end{array}$ \\
\end{tabular} & & & Control & 100 & \begin{tabular}{|l}
0.775 \\
$\mathrm{ab}$ \\
\end{tabular} & \begin{tabular}{|l|}
0.891 \\
$\mathrm{a}$ \\
\end{tabular} & \begin{tabular}{|l}
0.662 \\
$\mathrm{bc}$
\end{tabular} \\
\hline$\stackrel{0}{\frac{0}{0}}$ & 光 & 200 & & $\begin{array}{l}0.960 \\
\mathrm{a} \\
\end{array}$ & \begin{tabular}{|l}
0.889 \\
$\mathrm{a}$ \\
\end{tabular} & \begin{tabular}{|l}
0.602 \\
$\mathrm{~b}$
\end{tabular} & & & & 200 & \begin{tabular}{|l}
0.986 \\
$\mathrm{a}$ \\
\end{tabular} & $\begin{array}{l}0.892 \\
\mathrm{a} \\
\end{array}$ & \begin{tabular}{|l}
0.639 \\
$\mathrm{bc}$ \\
\end{tabular} \\
\hline 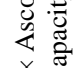 & & 0 & & \begin{tabular}{|l}
0.887 \\
$\mathrm{a}$
\end{tabular} & $\begin{array}{l}0.882 \\
\mathrm{a}\end{array}$ & $\begin{array}{l}0.565 \\
\mathrm{~b}\end{array}$ & & & & 0 & $\begin{array}{l}0.863 \\
\mathrm{a}\end{array}$ & $\begin{array}{l}0.872 \\
\mathrm{a}\end{array}$ & $\begin{array}{l}0.530 \\
\mathrm{c}\end{array}$ \\
\hline 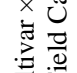 & $\begin{array}{l}0 \\
\Xi\end{array}$ & 100 & & $\begin{array}{l}0.826 \\
\mathrm{a}\end{array}$ & $\begin{array}{l}0.887 \\
\mathrm{a} \\
\end{array}$ & $\begin{array}{l}0.625 \\
\mathrm{~b}\end{array}$ & & & Corn & 100 & $\begin{array}{l}0.913 \\
\mathrm{a}\end{array}$ & $\begin{array}{l}0.875 \\
\mathrm{a} \\
\end{array}$ & $\begin{array}{l}0.576 \\
\text { bc }\end{array}$ \\
\hline$\vec{\exists} \bar{x}$ & $\frac{\pi}{\pi}$ & 200 & & $\begin{array}{l}0.985 \\
\mathrm{a}\end{array}$ & $\begin{array}{l}0.895 \\
\mathrm{a}\end{array}$ & $\begin{array}{l}0.650 \\
\mathrm{~b}\end{array}$ & & & & 200 & $\begin{array}{l}0.925 \\
\mathrm{a}\end{array}$ & $\begin{array}{l}0.897 \\
\mathrm{a}\end{array}$ & $\begin{array}{l}0.644 \\
\mathrm{bc}\end{array}$ \\
\hline & & & & & & & & & & 0 & $\begin{array}{l}0.893 \\
\mathrm{a}\end{array}$ & $\begin{array}{l}0.877 \\
\mathrm{a}\end{array}$ & $\begin{array}{l}0.522 \\
\text { c }\end{array}$ \\
\hline $\begin{array}{l}\text { Field } \\
\text { Capaci }\end{array}$ & & & & $\begin{array}{l}0.906 \\
\mathrm{a}\end{array}$ & $\begin{array}{l}0.885 \\
\mathrm{a}\end{array}$ & $\begin{array}{l}0.595 \\
b\end{array}$ & & & Sunflower & 100 & $\begin{array}{l}0.901 \\
\mathrm{a} \\
\end{array}$ & \begin{tabular}{|l}
0.889 \\
$\mathrm{a}$ \\
\end{tabular} & $\begin{array}{l}0.581 \\
\mathrm{bc}\end{array}$ \\
\hline & & & & & & & & & & 200 & $\begin{array}{l}0.980 \\
\mathrm{a}\end{array}$ & $\begin{array}{l}0.888 \\
\mathrm{a}\end{array}$ & $\begin{array}{l}0.595 \\
\text { bc }\end{array}$ \\
\hline
\end{tabular}


plant growth, which stimulated the increase of photosynthesis products, which were positively reflected in the production of carbohydrates.

This explanation was suggested with Choudhury (1993) [34] that found a positive effects of ASA on photosynthesis attributed to stabilizing and protecting the photosynthetic pigment and photosynthetic apparatus from oxidation. These results agreed with Hussein and Khursheed (2014) [25] foliar treatment of ASA which gave a significant increase of chlorophyll and carotenoids compared with control.

Relating to the interaction between ascorbic acid and field capacity there was a significant increase in total chlorophyll content when spraying at $200 \mathrm{ppm}$ and field capacity $85 \%$, while a significant decrease in carotenoid in field capacity $35 \%$ and non treated with ascorbic. According to the triple interaction (residues $\times$ ascorbic $\times$ field capacity) it was noted lowest value in corn residues not treated ascorbic and field capacity $35 \%$ in chlorophyll content, for carotenoid in sunflower residues, field capacity $35 \%$ and non acid treatment.

\section{Catalase and Peroxidase Activity}

Table $(6,7)$ show an increased activity of catalase and peroxidase in high moisture stress, adding water $35 \%$ of the field capacity gave the highest values in the activity of CAT and POD $(3.503,0.214 \%)$ compared to the field capacity $85 \%(2.929,0.129 \%)$ respectively. The increase of antioxidant enzyme was explained under water stress a defensive mechanism used by the plant to eliminate the toxic effect of reactive oxygen species (ROS) which is formatted as a result of an imbalance in electron transfer chain when stomata closure maintains to t moisture content of soil when exposed drought. The enzymes are responsible for decomposition and detoxification of $\mathrm{H}_{2} \mathrm{O}_{2}$ and convert it into water and oxygen [35]. Thus antioxidant enzymes role in protecting the plant from stress and maintain cell compound (Chloroplast, proteins and membranes) from oxidation by free radical [36]. These results agree with Mousi and Ibrahim (2018) [37] that indicated that water stress at 50\% from field capacity has given the highest values to the activity of POD and CAT of wheat.

Significant decrease in the activity of catalase enzyme of the control treatment (with residues) has been found, compared with corn and sunflower residues that were $(6.205,8.348 \%$ ) respectively, there was no significant increase in activity of peroxidase with the treatment of residues. Ibrahim et al. (2013) [33] explained the allelopathic effect to 3 extract $(3 \%, 5 \%, 10 \%)$ prepared from the leaves of genetically modified and non- modified corn on the activity of enzyme catalase in the wheat aqueous extracts enhanced the activity of catalase in wheat and maximum significant increase was recorded with lower aqueous extract of non-genetically modified as compared with control. Depending on the results that are obtained her be some allelochemical, might have increased the catalase activity in wheat. The same results were also obtained by Peng et al. (2004) [38] which the activity of the antioxidant enzyme was affected by allelopathic compounds. The results illustrate that the increased activity of both catalase and peroxidase was subject to different concentrations of ascorbic acid especially $200 \mathrm{ppm}$ compared with treatment without spray by $(20.22,37.78 \%)$ respectively. This result consistents with Behairy et al. (2012) [39] that mentioned that seeds fenugreek soaked in ascorbic acid had increased catalase activity in which seeds were not treated with ascorbic acid.

Plants protect cellular from the cytotoxic effect of active oxygen radicals by enzymatic mechanism (Such as catalase) as well as non- enzymatic mechanism (such as carotenoids and ascorbic acid) [40]. With respect to interaction between residues and field capacity the highest activity of the catalase and peroxidase in stressed condition (35\% field capacity) in sunflower residues compared to the other treatment. While the interaction of three factors (cultivar $\times$ residues $\times$ field capacity) 
was less value in antioxidant enzyme activity (CAT, POD) and for the treatment of cultivar IPA 99 in the field capacity $85 \%$ and without adding residues compared with other treatment.

Table (6) Effect of corn, sunflower residues and field capacity in catalase (observed activity) of wheat treated with ascorbic acid

\begin{tabular}{|c|c|c|c|c|c|c|c|c|c|c|c|c|c|}
\hline \multirow{2}{*}{ Cultivar } & & \multirow[b]{2}{*}{ Residues } & \multirow{2}{*}{$\begin{array}{l}\text { Ascorbic } \\
\text { acid }\end{array}$} & \multicolumn{3}{|c|}{ Field Capacity } & \multirow{2}{*}{$\begin{array}{l}\text { Cultivar } \\
\times \\
\text { Residues } \\
\times \\
\text { Ascorbic }\end{array}$} & \multirow{2}{*}{$\begin{array}{l}\text { Cultivar } \\
\times \\
\text { Residues }\end{array}$} & \multirow{2}{*}{$\begin{array}{l}\text { Cultivar } \\
\times \\
\text { Ascorbic }\end{array}$} & \multirow{2}{*}{$\begin{array}{l}\text { Residues } \\
\times \\
\text { Ascorbic }\end{array}$} & \multicolumn{3}{|c|}{$\begin{array}{l}\text { Cultivar } \times \text { Residues } \times \text { Field } \\
\text { Capacity }\end{array}$} \\
\hline & & & & 85 & 60 & 35 & & & & & 85 & 60 & 35 \\
\hline \multirow{9}{*}{\multicolumn{2}{|c|}{ IPA 99}} & \multirow{3}{*}{ Control } & 0 & $\begin{array}{l}2.410 \\
1\end{array}$ & $\begin{array}{l}2.520 \\
\mathrm{kl}\end{array}$ & $\begin{array}{l}3.120 \\
\mathrm{~b}-\mathrm{k}\end{array}$ & $\begin{array}{l}2.683 \\
\mathrm{H}\end{array}$ & \multirow{3}{*}{$\begin{array}{l}2.957 \\
\mathrm{c}\end{array}$} & $\begin{array}{l}2.807 \\
\mathrm{~d}\end{array}$ & $\begin{array}{l}2.717 \\
\mathrm{e}\end{array}$ & 2.780 & $\begin{array}{l}2.847 \\
\mathrm{fg}\end{array}$ & $\begin{array}{l}3.243 \\
c-e\end{array}$ \\
\hline & & & 100 & $\begin{array}{l}2.820 \\
\mathrm{f}-1\end{array}$ & $\begin{array}{l}2.853 \\
\mathrm{f}-1\end{array}$ & $\begin{array}{l}3.200 \\
b-k\end{array}$ & $\begin{array}{l}2.957 \\
e-h\end{array}$ & & $\begin{array}{l}3.056 \\
\mathrm{bc}\end{array}$ & $\begin{array}{l}3.020 \\
\mathrm{~d}\end{array}$ & & & \\
\hline & & & 200 & $\begin{array}{l}3.110 \\
b-k\end{array}$ & $\begin{array}{l}3.170 \\
b-k\end{array}$ & $\begin{array}{l}3.410 \\
\mathrm{a}-\mathrm{g}\end{array}$ & $\begin{array}{l}3.230 \\
b-e\end{array}$ & & \begin{tabular}{|l}
3.415 \\
$\mathrm{a}$
\end{tabular} & $\begin{array}{l}3.286 \\
\mathrm{bc}\end{array}$ & & & \\
\hline & & \multirow{3}{*}{ Corn } & 0 & $\begin{array}{l}2.610 \\
\mathrm{j}-\mathrm{k}\end{array}$ & $\begin{array}{l}2.660 \\
i-1\end{array}$ & $\begin{array}{l}3.210 \\
\mathrm{~b}-\mathrm{k}\end{array}$ & $\begin{array}{l}2.826 \\
\mathrm{f}-\mathrm{h}\end{array}$ & \multirow{3}{*}{$\begin{array}{l}3.108 \\
\mathrm{bc}\end{array}$} & & $\begin{array}{l}2.982 \\
\mathrm{~d}\end{array}$ & $\begin{array}{l}2.916 \\
e-g\end{array}$ & $\begin{array}{l}2.996 \\
\mathrm{~d}-\mathrm{g}\end{array}$ & $\begin{array}{l}3.412 \\
\mathrm{a}-\mathrm{c}\end{array}$ \\
\hline & & & 100 & $\begin{array}{l}2.920 \\
\mathrm{~d}-1\end{array}$ & $\begin{array}{l}2.960 \\
\mathrm{~d}-1\end{array}$ & $\begin{array}{l}3.316 \\
\mathrm{a}-\mathrm{i}\end{array}$ & $\begin{array}{l}3.065 \\
\mathrm{c}-\mathrm{g}\end{array}$ & & & \begin{tabular}{|l|}
3.133 \\
$\mathrm{~cd}$
\end{tabular} & & & \\
\hline & & & 200 & $\begin{array}{l}3.220 \\
\mathrm{a}-\mathrm{i}\end{array}$ & $\begin{array}{l}3.370 \\
\text { a-h }\end{array}$ & $\begin{array}{l}3.710 \\
\text { a-c }\end{array}$ & $\begin{array}{l}3.433 \\
\text { a-c }\end{array}$ & & & $\begin{array}{l}3.506 \\
\mathrm{ab}\end{array}$ & & & \\
\hline & & \multirow{3}{*}{ Sunflower } & 0 & $\begin{array}{l}2.660 \\
\mathrm{i}-\mathrm{k}\end{array}$ & $\begin{array}{l}2.660 \\
i-k\end{array}$ & $\begin{array}{l}3.320 \\
\mathrm{a}-\mathrm{i}\end{array}$ & $\begin{array}{l}2.913 \\
\text { e-h }\end{array}$ & \multirow{3}{*}{$\begin{array}{l}3.210 \\
\mathrm{ab}\end{array}$} & & $\begin{array}{l}2.963 \\
\mathrm{~d}\end{array}$ & $\begin{array}{l}2.885 \\
\mathrm{e}-\mathrm{g}\end{array}$ & $\begin{array}{l}3.140 \\
\mathrm{c}-\mathrm{g}\end{array}$ & $\begin{array}{l}3.616 \\
\mathrm{ab}\end{array}$ \\
\hline & & & 100 & $\begin{array}{l}2.656 \\
\mathrm{i}-\mathrm{k}\end{array}$ & $\begin{array}{l}3.170 \\
b-k\end{array}$ & $\begin{array}{l}3.610 \\
a-d\end{array}$ & $\begin{array}{l}3.145 \\
c-f\end{array}$ & & & $\begin{array}{l}3.263 \\
\mathrm{c} \\
\end{array}$ & & & \\
\hline & & & 200 & $\begin{array}{l}3.340 \\
\mathrm{a}-\mathrm{i}\end{array}$ & $\begin{array}{l}3.490 \\
a-f\end{array}$ & $\begin{array}{l}3.920 \\
\mathrm{a}\end{array}$ & $\begin{array}{l}3.583 \\
\mathrm{Ab}\end{array}$ & & & $\begin{array}{l}3.621 \\
\mathrm{a} \\
\end{array}$ & & & \\
\hline \multirow{9}{*}{ Sham 6} & & \multirow{3}{*}{ Control } & 0 & $\begin{array}{l}2.516 \\
\mathrm{kl}\end{array}$ & $\begin{array}{l}2.530 \\
\mathrm{j}-1\end{array}$ & $\begin{array}{l}3.210 \\
\text { b-k }\end{array}$ & $\begin{array}{l}2.752 \\
\text { Gh }\end{array}$ & \multirow{3}{*}{$\begin{array}{l}3.059 \\
\text { bc }\end{array}$} & $\begin{array}{l}2.967 \\
\mathrm{~cd}\end{array}$ & & $\begin{array}{l}2.878 \\
\mathrm{e}-\mathrm{g}\end{array}$ & $\begin{array}{l}2.963 \\
\mathrm{~d}-\mathrm{g}\end{array}$ & $\begin{array}{l}3.336 \\
\text { b-d }\end{array}$ \\
\hline & & & 100 & $\begin{array}{l}2.910 \\
\mathrm{e}-1\end{array}$ & $\begin{array}{l}3.030 \\
\mathrm{c}-1\end{array}$ & $\begin{array}{l}3.310 \\
\mathrm{a}-\mathrm{i}\end{array}$ & $\begin{array}{l}3.083 \\
\mathrm{c}-\mathrm{g}\end{array}$ & & $\begin{array}{l}3.222 \\
\mathrm{~b}\end{array}$ & & & & \\
\hline & & & 200 & $\begin{array}{l}3.210 \\
b-k\end{array}$ & $\begin{array}{l}3.330 \\
\mathrm{a}-\mathrm{i}\end{array}$ & $\begin{array}{l}3.490 \\
\text { a-e }\end{array}$ & $\begin{array}{l}3.343 \\
a-d\end{array}$ & & \begin{tabular}{|l|}
3.527 \\
$\mathrm{a}$
\end{tabular} & & & & \\
\hline & & \multirow{3}{*}{ Corn } & 0 & $\begin{array}{l}2.813 \\
\mathrm{f}-1 \\
\end{array}$ & $\begin{array}{l}2.890 \\
\mathrm{e}-1\end{array}$ & $\begin{array}{l}3.710 \\
a-c\end{array}$ & $\begin{array}{l}3.137 \\
\mathrm{c}-\mathrm{f}\end{array}$ & \multirow{3}{*}{$\begin{array}{l}3.306 \\
\mathrm{a}\end{array}$} & & & $\begin{array}{l}3.056 \\
\mathrm{c}-\mathrm{g}\end{array}$ & $\begin{array}{l}3.193 \\
c-f\end{array}$ & $\begin{array}{l}3.670 \\
\mathrm{ab}\end{array}$ \\
\hline & & & 100 & $\begin{array}{l}2.986 \\
\text { d-1 }\end{array}$ & $\begin{array}{l}3.110 \\
b-k\end{array}$ & $\begin{array}{l}3.510 \\
a-f\end{array}$ & $\begin{array}{l}3.202 \\
\text { c-e }\end{array}$ & & & & & & \\
\hline & & & 200 & $\begin{array}{l}3.370 \\
a-h\end{array}$ & $\begin{array}{l}3.580 \\
\text { a-e }\end{array}$ & $\begin{array}{l}3.790 \\
\mathrm{ab}\end{array}$ & $\begin{array}{l}3.580 \\
\mathrm{Ab}\end{array}$ & & & & & & \\
\hline & & \multirow{3}{*}{ Sunflower } & 0 & $\begin{array}{l}2.710 \\
h-1\end{array}$ & $\begin{array}{l}2.820 \\
\mathrm{f}-1 \\
\end{array}$ & $\begin{array}{l}3.510 \\
\text { a-f }\end{array}$ & $\begin{array}{l}3.013 \\
\mathrm{~d}-\mathrm{g}\end{array}$ & \multirow{3}{*}{$\begin{array}{l}3.351 \\
\mathrm{a}\end{array}$} & & & $\begin{array}{l}3.058 \\
\mathrm{c}-\mathrm{g}\end{array}$ & $\begin{array}{l}3.256 \\
\text { c-e }\end{array}$ & $\begin{array}{l}3.740 \\
\mathrm{a}\end{array}$ \\
\hline & & & 100 & $\begin{array}{l}3.016 \\
\mathrm{c}-1\end{array}$ & $\begin{array}{l}3.380 \\
a-h\end{array}$ & $\begin{array}{l}3.750 \\
\mathrm{ab}\end{array}$ & $\begin{array}{l}3.382 \\
a-d\end{array}$ & & & & & & \\
\hline & & & 200 & $\begin{array}{l}3.450 \\
\mathrm{a}-\mathrm{g} \\
\end{array}$ & \begin{tabular}{|l}
3.570 \\
a-e
\end{tabular} & $\begin{array}{l}3.960 \\
\mathrm{a} \\
\end{array}$ & $\begin{array}{l}3.660 \\
\mathrm{~A} \\
\end{array}$ & & & & & & \\
\hline & & & & & & & & Cultivar & Residues & Ascorbic & $\begin{array}{l}\text { Residu } \\
\text { Aserob }\end{array}$ & $\times$ Fiel & Capacity $\times$ \\
\hline \multirow{2}{*}{\multicolumn{2}{|c|}{$\begin{array}{l}\text { Cultivar } \\
\times \\
\text { Field } \\
\text { Capacity }\end{array}$}} & \multicolumn{2}{|l|}{ IPA } & $\begin{array}{l}2.860 \\
\mathrm{c}\end{array}$ & $\begin{array}{l}2.994 \\
\text { bc }\end{array}$ & $\begin{array}{l}3.424 \\
\mathrm{a}\end{array}$ & & $\begin{array}{l}3.093 \\
\mathrm{~b}\end{array}$ & & & & & \\
\hline & & \multicolumn{2}{|l|}{ Sham 6} & $\begin{array}{l}2.996 \\
\mathrm{c}\end{array}$ & $\begin{array}{l}3.137 \\
\mathrm{~b}\end{array}$ & \begin{tabular}{|l}
3.582 \\
$\mathrm{a}$
\end{tabular} & & $\begin{array}{l}3.239 \\
\mathrm{a}\end{array}$ & & & & & \\
\hline Residue & & Con-T & & $\begin{array}{l}2.829 \\
\mathrm{e}\end{array}$ & $\begin{array}{l}2.905 \\
\mathrm{de}\end{array}$ & $\begin{array}{l}3.290 \\
b\end{array}$ & & & $\begin{array}{l}3.008 \\
\mathrm{~b}\end{array}$ & & & & \\
\hline $\begin{array}{l}\text { Field } \\
\text { Field }\end{array}$ & & Corn & & $\begin{array}{l}2.980 \\
\text { c-e }\end{array}$ & $\begin{array}{l}3.095 \\
\text { b-d }\end{array}$ & $\begin{array}{l}3.541 \\
\mathrm{a}\end{array}$ & & & \begin{tabular}{|l}
3.207 \\
$\mathrm{a}$
\end{tabular} & & & & \\
\hline Capacit & & Sunflower & & $\begin{array}{l}2.972 \\
\mathrm{c}-\mathrm{e}\end{array}$ & $\begin{array}{l}3.198 \\
\text { bc }\end{array}$ & $\begin{array}{l}3.678 \\
\mathrm{a}\end{array}$ & & & \begin{tabular}{|l|}
3.282 \\
$\mathrm{a}$ \\
\end{tabular} & & & & \\
\hline Ascorbi & & 0 & & $\begin{array}{l}2.620 \\
f\end{array}$ & $\begin{array}{l}2.696 \\
\text { ef }\end{array}$ & $\begin{array}{l}3.346 \\
b\end{array}$ & & & & $\begin{array}{l}2.827 \\
\mathrm{c}\end{array}$ & & & \\
\hline $\begin{array}{l}\times \\
\text { Field }\end{array}$ & & 100 & & $\begin{array}{l}2.885 \\
\text { de }\end{array}$ & $\begin{array}{l}3.083 \\
\text { cd }\end{array}$ & $\begin{array}{l}3.449 \\
b\end{array}$ & & & & $\begin{array}{l}3.139 \\
\mathrm{~b}\end{array}$ & & & \\
\hline Capacit & & 200 & & $\begin{array}{l}3.283 \\
\text { bc }\end{array}$ & $\begin{array}{l}3.418 \\
\mathrm{~b}\end{array}$ & $\begin{array}{l}3.713 \\
\mathrm{a}\end{array}$ & & & & $\begin{array}{l}3.471 \\
\mathrm{a} \\
\end{array}$ & & & \\
\hline$x$ & & 0 & & $\begin{array}{l}2.560 \\
\mathrm{~g}\end{array}$ & $\begin{array}{l}2.646 \\
\mathrm{fg}\end{array}$ & $\begin{array}{l}3.216 \\
\text { c-e }\end{array}$ & & & & 0 & $\begin{array}{l}2.463 \\
1\end{array}$ & $\begin{array}{l}2.525 \\
\mathrm{kl}\end{array}$ & $\begin{array}{l}3.165 \\
\mathrm{~d}-\mathrm{i}\end{array}$ \\
\hline$=.00$ & જे & 100 & & $\begin{array}{l}2.798 \\
\mathrm{fg}\end{array}$ & $\begin{array}{l}2.994 \\
\text { d-f }\end{array}$ & $\begin{array}{l}3.375 \\
\mathrm{bc}\end{array}$ & & & Control & 100 & $\begin{array}{l}2.865 \\
\text { h-1 }\end{array}$ & $\begin{array}{l}2.941 \\
\mathrm{~g}-\mathrm{j}\end{array}$ & $\begin{array}{l}3.255 \\
\mathrm{c}-\mathrm{h}\end{array}$ \\
\hline 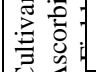 & $\underset{\Xi}{\varrho}$ & 200 & & $\begin{array}{l}3.223 \\
\mathrm{c}-\mathrm{e}\end{array}$ & $\begin{array}{l}3.343 \\
\text { b-d }\end{array}$ & $\begin{array}{l}3.680 \\
\mathrm{ab}\end{array}$ & & & & 200 & $\begin{array}{l}3.160 \\
d-i\end{array}$ & $\begin{array}{l}3.250 \\
\mathrm{c}-\mathrm{h}\end{array}$ & $\begin{array}{l}3.450 \\
b-e\end{array}$ \\
\hline & क- & 0 & & 2.680 & 2.746 & 3.476 & & & Corn & 0 & 2.711 & 2.775 & 3.460 \\
\hline
\end{tabular}


Journal of Education and Science (ISSN 1812-125X), Vol: 29, No: 2, 2020 (260-278)

\begin{tabular}{|c|c|c|c|c|c|c|c|c|c|}
\hline & & $\mathrm{fg}$ & fg & $\mathrm{a}-\mathrm{c}$ & & & i-1 & i-1 & $\mathrm{b}-\mathrm{e}$ \\
\hline & 100 & $\begin{array}{l}2.970 \\
\text { ef }\end{array}$ & $\begin{array}{l}3.173 \\
\text { c-e }\end{array}$ & $\begin{array}{l}3.523 \\
\text { a-c }\end{array}$ & & 100 & $\begin{array}{l}2.983 \\
\mathrm{f}-\mathrm{k}\end{array}$ & $\begin{array}{l}3.035 \\
e-j\end{array}$ & $\begin{array}{l}3.413 \\
\text { b-f }\end{array}$ \\
\hline & 200 & $\begin{array}{l}3.343 \\
\text { b-d }\end{array}$ & $\begin{array}{l}3.493 \\
a-c\end{array}$ & $\begin{array}{l}3.746 \\
\text { A }\end{array}$ & & 200 & $\begin{array}{l}3.295 \\
\text { b-h }\end{array}$ & $\begin{array}{l}3.475 \\
\text { b-e }\end{array}$ & $\begin{array}{l}3.750 \\
\mathrm{ab}\end{array}$ \\
\hline \multirow{3}{*}{$\begin{array}{l}\text { Field } \\
\text { Capacity }\end{array}$} & & \multirow{3}{*}{$\begin{array}{l}2.929 \\
\mathrm{c}\end{array}$} & \multirow{3}{*}{$\begin{array}{l}3.066 \\
b\end{array}$} & \multirow{3}{*}{$\begin{array}{l}3.503 \\
\mathrm{~A}\end{array}$} & \multirow{3}{*}{ Sunflower } & 0 & $\begin{array}{l}2.685 \\
j-1\end{array}$ & $\begin{array}{l}2.790 \\
i-1\end{array}$ & $\begin{array}{l}3.415 \\
\text { b-f }\end{array}$ \\
\hline & & & & & & 100 & $\begin{array}{l}2.836 \\
\mathrm{n}-1\end{array}$ & $\begin{array}{l}3.275 \\
\text { b-h }\end{array}$ & $\begin{array}{l}3.680 \\
a-c\end{array}$ \\
\hline & & & & & & 200 & $\begin{array}{l}3.395 \\
\text { b-g }\end{array}$ & $\begin{array}{l}3.530 \\
\text { a-d }\end{array}$ & $\begin{array}{l}3.940 \\
\mathrm{a}\end{array}$ \\
\hline
\end{tabular}

Table (7) Effect of corn, sunflower residues and field capacity in peroxidase $(\mu \mathrm{mol} / \mathrm{ml})$ of wheat treated with ascorbic acid

\begin{tabular}{|c|c|c|c|c|c|c|c|c|c|c|c|c|}
\hline \multirow[b]{2}{*}{ Cultivar } & \multirow[b]{2}{*}{ Residues } & \multirow{2}{*}{$\begin{array}{l}\text { Ascorbic } \\
\text { acid }\end{array}$} & \multicolumn{3}{|c|}{ Field Capacity } & \multirow{2}{*}{$\begin{array}{l}\text { Cultivar } \\
\times \\
\text { Residues } \\
\times \\
\text { Ascorbic }\end{array}$} & \multirow{2}{*}{$\begin{array}{l}\text { Cultivar } \\
\times \\
\text { Residues }\end{array}$} & \multirow{2}{*}{$\begin{array}{l}\text { Cultivar } \\
\times \\
\text { Ascorbic }\end{array}$} & \multirow{2}{*}{$\begin{array}{l}\text { Residues } \\
\times \\
\text { Ascorbic }\end{array}$} & \multicolumn{3}{|c|}{$\begin{array}{l}\text { Cultivar } \times \text { Residues } \times \text { Field } \\
\text { Capacity }\end{array}$} \\
\hline & & & 85 & 60 & 35 & & & & & 85 & 60 & 35 \\
\hline \multirow{9}{*}{ IPA 99} & \multirow{3}{*}{ Control } & 0 & $\begin{array}{l}0.092 \\
\mathrm{~g}\end{array}$ & $\begin{array}{l}0.121 \\
\mathrm{~d}-\mathrm{g}\end{array}$ & $\begin{array}{l}0.212 \\
\mathrm{a}-\mathrm{e}\end{array}$ & $\begin{array}{l}0.141 \\
b-d\end{array}$ & \multirow{3}{*}{$\begin{array}{l}0.153 \\
b\end{array}$} & $\begin{array}{l}0.140 \\
\mathrm{~B}\end{array}$ & $\begin{array}{l}0.129 \\
\mathrm{~d}\end{array}$ & $\begin{array}{l}0.101 \\
\mathrm{~g}\end{array}$ & $\begin{array}{l}0.144 \\
\mathrm{~d}-\mathrm{g}\end{array}$ & $\begin{array}{l}0.213 \\
\mathrm{a}-\mathrm{c}\end{array}$ \\
\hline & & 100 & $\begin{array}{l}0.101 \\
\mathrm{fg}\end{array}$ & $\begin{array}{l}0.141 \\
b-g\end{array}$ & $\begin{array}{l}0.211 \\
\text { a-e }\end{array}$ & $\begin{array}{l}0.151 \\
a-d\end{array}$ & & $\begin{array}{l}0.161 \\
\mathrm{Ab}\end{array}$ & $\begin{array}{l}0.171 \\
\mathrm{a}-\mathrm{c}\end{array}$ & & & \\
\hline & & 200 & $\begin{array}{l}0.112 \\
\mathrm{e}-\mathrm{g}\end{array}$ & $\begin{array}{l}0.171 \\
\mathrm{a}-\mathrm{g}\end{array}$ & $\begin{array}{l}0.218 \\
\text { a-e }\end{array}$ & $\begin{array}{l}0.167 \\
a-d\end{array}$ & & $\begin{array}{l}0.180 \\
\mathrm{~A}\end{array}$ & $\begin{array}{l}0.171 \\
\mathrm{a}-\mathrm{c}\end{array}$ & & & \\
\hline & \multirow{3}{*}{ Corn } & 0 & $\begin{array}{l}0.093 \\
\mathrm{~g}\end{array}$ & $\begin{array}{l}0.123 \\
\text { d-g }\end{array}$ & $\begin{array}{l}0.194 \\
\mathrm{a}-\mathrm{g}\end{array}$ & $\begin{array}{l}0.136 \\
\mathrm{~cd}\end{array}$ & \multirow{3}{*}{$\begin{array}{l}0.161 \\
\mathrm{ab}\end{array}$} & & $\begin{array}{l}0.137 \\
\text { cd }\end{array}$ & $\begin{array}{l}0.112 \\
\mathrm{fg}\end{array}$ & $\begin{array}{l}0.159 \\
\mathrm{c}-\mathrm{f}\end{array}$ & $\begin{array}{l}0.211 \\
\mathrm{a}-\mathrm{c}\end{array}$ \\
\hline & & 100 & $\begin{array}{l}0.112 \\
\mathrm{e}-\mathrm{g}\end{array}$ & $\begin{array}{l}0.161 \\
\mathrm{a}-\mathrm{g}\end{array}$ & $\begin{array}{l}0.219 \\
\text { a-e }\end{array}$ & $\begin{array}{l}0.164 \\
a-d\end{array}$ & & & $\begin{array}{l}0.170 \\
\mathrm{a}-\mathrm{c}\end{array}$ & & & \\
\hline & & 200 & $\begin{array}{l}0.131 \\
\mathrm{c}-\mathrm{g}\end{array}$ & $\begin{array}{l}0.195 \\
\mathrm{a}-\mathrm{g}\end{array}$ & $\begin{array}{l}0.221 \\
\text { a-d }\end{array}$ & $\begin{array}{l}0.182 \\
\mathrm{a}-\mathrm{c}\end{array}$ & & & $\begin{array}{l}0.186 \\
\mathrm{ab}\end{array}$ & & & \\
\hline & \multirow{3}{*}{ Sunflower } & 0 & $\begin{array}{l}0.097 \\
\text { fg }\end{array}$ & $\begin{array}{l}0.135 \\
\mathrm{c}-\mathrm{g}\end{array}$ & $\begin{array}{l}0.199 \\
\mathrm{a}-\mathrm{g}\end{array}$ & $\begin{array}{l}0.143 \\
b-d\end{array}$ & \multirow{3}{*}{$\begin{array}{l}0.168 \\
\mathrm{ab}\end{array}$} & & $\begin{array}{l}0.148 \\
\text { b-d }\end{array}$ & $\begin{array}{l}0.119 \\
\mathrm{e}-\mathrm{g}\end{array}$ & $\begin{array}{l}0.167 \\
\text { b-f }\end{array}$ & $\begin{array}{l}0.219 \\
\mathrm{a}\end{array}$ \\
\hline & & 100 & $\begin{array}{l}0.121 \\
\mathrm{~d}-\mathrm{g}\end{array}$ & $\begin{array}{l}0.169 \\
\mathrm{a}-\mathrm{g}\end{array}$ & $\begin{array}{l}0.221 \\
\mathrm{a}-\mathrm{d}\end{array}$ & $\begin{array}{l}0.170 \\
\text { a-d }\end{array}$ & & & $\begin{array}{l}0.184 \\
a b\end{array}$ & & & \\
\hline & & 200 & $\begin{array}{l}0.141 \\
b-g\end{array}$ & $\begin{array}{l}0.198 \\
\text { a-g }\end{array}$ & $\begin{array}{l}0.237 \\
\text { a-c }\end{array}$ & $\begin{array}{l}0.192 \\
a-c\end{array}$ & & & \begin{tabular}{|l}
0.200 \\
$\mathrm{a}$
\end{tabular} & & & \\
\hline \multirow{10}{*}{ Sham 6} & \multirow{3}{*}{ Control } & 0 & $\begin{array}{l}0.097 \\
\mathrm{fg}\end{array}$ & $\begin{array}{l}0.128 \\
\mathrm{~d}-\mathrm{g}\end{array}$ & $\begin{array}{l}0.128 \\
\text { d-g }\end{array}$ & $\begin{array}{l}0.117 \\
\mathrm{~d}\end{array}$ & \multirow{3}{*}{$\begin{array}{l}0.162 \\
\mathrm{ab}\end{array}$} & $\begin{array}{l}0.136 \\
\mathrm{~A}\end{array}$ & & $\begin{array}{l}0.146 \\
\mathrm{~d}-\mathrm{g}\end{array}$ & $\begin{array}{l}0.152 \\
\mathrm{~d}-\mathrm{g}\end{array}$ & $\begin{array}{l}0.188 \\
\text { a-d }\end{array}$ \\
\hline & & 100 & $\begin{array}{l}0.212 \\
\text { a-e }\end{array}$ & $\begin{array}{l}0.149 \\
\mathrm{a}-\mathrm{g}\end{array}$ & $\begin{array}{l}0.217 \\
\text { a-e }\end{array}$ & $\begin{array}{l}0.192 \\
a-c\end{array}$ & & $\begin{array}{l}0.188 \\
\mathrm{~A}\end{array}$ & & & & \\
\hline & & 200 & $\begin{array}{l}0.131 \\
\mathrm{c}-\mathrm{g}\end{array}$ & $\begin{array}{l}0.179 \\
\mathrm{a}-\mathrm{g}\end{array}$ & $\begin{array}{l}0.219 \\
\text { a-e }\end{array}$ & $\begin{array}{l}0.176 \\
a-d\end{array}$ & & $\begin{array}{l}0.192 \\
\mathrm{~A}\end{array}$ & & & & \\
\hline & \multirow{3}{*}{ Corn } & 0 & $\begin{array}{l}0.096 \\
\text { fg }\end{array}$ & $\begin{array}{l}0.125 \\
\mathrm{~d}-\mathrm{g} \\
\end{array}$ & $\begin{array}{l}0.195 \\
\mathrm{a}-\mathrm{g} \\
\end{array}$ & $\begin{array}{l}0.138 \\
b-d\end{array}$ & \multirow{3}{*}{$\begin{array}{l}0.168 \\
a b\end{array}$} & & & $\begin{array}{l}0.124 \\
\mathrm{e}-\mathrm{g}\end{array}$ & $\begin{array}{l}0.166 \\
b-f\end{array}$ & $\begin{array}{l}0.215 \\
a-c\end{array}$ \\
\hline & & 100 & $\begin{array}{l}0.131 \\
\mathrm{c}-\mathrm{g}\end{array}$ & \begin{tabular}{|l|}
0.177 \\
$\mathrm{a}-\mathrm{g}$
\end{tabular} & $\begin{array}{l}0.221 \\
\text { a-e } \\
\end{array}$ & $\begin{array}{l}0.176 \\
\text { a-d } \\
\end{array}$ & & & & & & \\
\hline & & 200 & $\begin{array}{l}0.146 \\
\mathrm{a}-\mathrm{g} \\
\end{array}$ & \begin{tabular}{|l}
0.198 \\
$\mathrm{a}-\mathrm{g}$ \\
\end{tabular} & \begin{tabular}{|l|}
0.229 \\
a-d
\end{tabular} & \begin{tabular}{|l}
0.191 \\
$\mathrm{a}-\mathrm{c}$
\end{tabular} & & & & & & \\
\hline & \multirow{3}{*}{ Sunflower } & 0 & $\begin{array}{l}0.099 \\
\text { fg }\end{array}$ & $\begin{array}{l}0.139 \\
b-g\end{array}$ & $\begin{array}{l}0.221 \\
a-d\end{array}$ & $\begin{array}{l}0.153 \\
a-d\end{array}$ & \multirow{3}{*}{$\begin{array}{l}0.186 \\
\mathrm{a}\end{array}$} & & & $\begin{array}{l}0.145 \\
d-g\end{array}$ & $\begin{array}{l}0.176 \\
b-e\end{array}$ & $\begin{array}{l}0.237 \\
\mathrm{a}\end{array}$ \\
\hline & & 100 & $\begin{array}{l}0.161 \\
\mathrm{a}-\mathrm{g}\end{array}$ & $\begin{array}{l}0.189 \\
\mathrm{a}-\mathrm{g}\end{array}$ & $\begin{array}{l}0.243 \\
\mathrm{ab}\end{array}$ & $\begin{array}{l}0.197 \\
\mathrm{ab}\end{array}$ & & & & & & \\
\hline & & 200 & $\begin{array}{l}0.176 \\
a-g\end{array}$ & $\begin{array}{l}0.201 \\
\text { a-f }\end{array}$ & $\begin{array}{l}0.249 \\
\mathrm{a}\end{array}$ & \begin{tabular}{|l}
0.208 \\
$\mathrm{a}$
\end{tabular} & & & & & & \\
\hline & & & & & & & Cultivar & Residues & Ascorbic & $\begin{array}{l}\text { Residu } \\
\text { Aserol }\end{array}$ & $\times$ Fiel & Zapacity $\times$ \\
\hline \multirow{2}{*}{$\begin{array}{l}\text { Cultivar } \\
\times \\
\text { Field } \\
\text { Capacity }\end{array}$} & \multicolumn{2}{|l|}{ IPA } & $\begin{array}{l}0.111 \\
\mathrm{c}\end{array}$ & $\begin{array}{l}0.157 \\
\mathrm{~b}\end{array}$ & $\begin{array}{l}0.214 \\
\mathrm{a}\end{array}$ & & $\begin{array}{l}0.160 \\
\mathrm{a}\end{array}$ & & & & & \\
\hline & \multicolumn{2}{|l|}{ Sham 6} & $\begin{array}{l}0.138 \\
\mathrm{bc}\end{array}$ & $\begin{array}{l}0.165 \\
\mathrm{~b}\end{array}$ & $\begin{array}{l}0.213 \\
\mathrm{a}\end{array}$ & & $\begin{array}{l}0.172 \\
\mathrm{a}\end{array}$ & & & & & \\
\hline \multirow{3}{*}{$\begin{array}{l}\text { Residues } \\
\times \\
\text { Field } \\
\text { Capacity }\end{array}$} & \multicolumn{2}{|l|}{ Con-T } & $\begin{array}{l}0.124 \\
\mathrm{e}\end{array}$ & $\begin{array}{l}0.148 \\
\text { c-e }\end{array}$ & $\begin{array}{l}0.200 \\
a b\end{array}$ & & & $\begin{array}{l}0.157 \\
\mathrm{~A}\end{array}$ & & & & \\
\hline & Corn & & $\begin{array}{l}0.118 \\
\mathrm{e}\end{array}$ & $\begin{array}{l}0.163 \\
\mathrm{bc}\end{array}$ & $\begin{array}{l}0.213 \\
\mathrm{a}\end{array}$ & & & $\begin{array}{l}0.164 \\
\mathrm{~A}\end{array}$ & & & & \\
\hline & Sunflower & & \begin{tabular}{|l}
0.132 \\
de
\end{tabular} & \begin{tabular}{|l}
0.171 \\
bc \\
\end{tabular} & $\begin{array}{l}0.228 \\
\mathrm{a} \\
\end{array}$ & & & \begin{tabular}{|l|}
0.177 \\
$\mathrm{~A}$ \\
\end{tabular} & & & & \\
\hline Ascorbic & 0 & & $\begin{array}{l}0.095 \\
\mathrm{e}\end{array}$ & \begin{tabular}{|l}
0.128 \\
de
\end{tabular} & $\begin{array}{l}0.191 \\
\text { bc }\end{array}$ & & & & $\begin{array}{l}0.138 \\
\mathrm{~b} \\
\end{array}$ & & & \\
\hline $\begin{array}{l}X \\
\text { Field } \\
\text { Canacity }\end{array}$ & 100 & & $\begin{array}{l}0.139 \\
\mathrm{~d}\end{array}$ & $\begin{array}{l}0.164 \\
\text { cd }\end{array}$ & $\begin{array}{l}0.222 \\
a b\end{array}$ & & & & \begin{tabular}{|l|}
0.175 \\
$\mathrm{a}$ \\
\end{tabular} & & & \\
\hline Capacily & 200 & & 0.139 & 0.190 & 0.228 & & & & 0.186 & & & \\
\hline
\end{tabular}




\begin{tabular}{|c|c|c|c|c|c|c|c|c|c|c|}
\hline & & & $\mathrm{d}$ & $b c$ & $\mathrm{a}$ & & $\mathrm{a}$ & & & \\
\hline \multirow{6}{*}{ 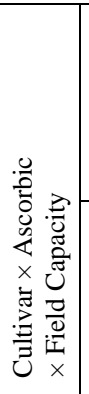 } & \multirow{3}{*}{$\begin{array}{l}2 \\
\overleftrightarrow{\leftrightarrows} \\
\Leftrightarrow\end{array}$} & 0 & $\begin{array}{l}0.940 \\
\mathrm{~h}\end{array}$ & $\begin{array}{l}0.126 \\
\text { e-h }\end{array}$ & $\begin{array}{l}0.201 \\
\text { a-d }\end{array}$ & \multirow{3}{*}{ Control } & 0 & $\begin{array}{l}0.945 \\
\mathrm{j}\end{array}$ & $\begin{array}{l}0.124 \\
\text { g-j }\end{array}$ & $\begin{array}{l}0.170 \\
\text { b-i }\end{array}$ \\
\hline & & 100 & $\begin{array}{l}0.111 \\
\text { f-h }\end{array}$ & $\begin{array}{l}0.157 \\
\text { df }\end{array}$ & $\begin{array}{l}0.217 \\
\text { a-c }\end{array}$ & & 100 & $\begin{array}{l}0.156 \\
c-j\end{array}$ & $\begin{array}{l}0.145 \\
d-j\end{array}$ & \begin{tabular}{|l|}
0.240 \\
a-d
\end{tabular} \\
\hline & & 200 & $\begin{array}{l}0.128 \\
\text { e-h }\end{array}$ & $\begin{array}{l}0.188 \\
\text { a-d }\end{array}$ & $\begin{array}{l}0.225 \\
\mathrm{ab}\end{array}$ & & 200 & $\begin{array}{l}0.121 \\
h-j\end{array}$ & $\begin{array}{l}0.175 \\
\text { a-h }\end{array}$ & $\begin{array}{l}0.218 \\
\text { a-c }\end{array}$ \\
\hline & \multirow{3}{*}{ 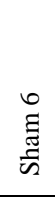 } & 0 & $\begin{array}{l}0.097 \\
\text { gh }\end{array}$ & $\begin{array}{l}0.130 \\
\text { e-h }\end{array}$ & \begin{tabular}{|l|}
0.181 \\
a-e
\end{tabular} & \multirow{3}{*}{ Corn } & 0 & $\begin{array}{l}0.094 \\
\mathrm{j}\end{array}$ & \begin{tabular}{|l|}
0.124 \\
$a-j$
\end{tabular} & $\begin{array}{l}0.194 \\
\text { a-g }\end{array}$ \\
\hline & & 100 & $\begin{array}{l}0.168 \\
\text { c-e }\end{array}$ & $\begin{array}{l}0.171 \\
\text { b-e }\end{array}$ & $\begin{array}{l}0.227 \\
\mathrm{ab}\end{array}$ & & 100 & $\begin{array}{l}0.121 \\
h-j\end{array}$ & $\begin{array}{l}0.169 \\
\text { b-i }\end{array}$ & $\begin{array}{l}0.220 \\
\text { a-c }\end{array}$ \\
\hline & & 200 & $\begin{array}{l}0.151 \\
\text { d-g }\end{array}$ & $\begin{array}{l}0.192 \\
\text { a-d }\end{array}$ & \begin{tabular}{|l|}
0.232 \\
$\mathrm{a}$
\end{tabular} & & 200 & $\begin{array}{l}0.138 \\
e-j\end{array}$ & $\begin{array}{l}0.196 \\
\text { a-g }\end{array}$ & $\begin{array}{l}0.225 \\
\text { a-c }\end{array}$ \\
\hline \multirow{3}{*}{\multicolumn{2}{|c|}{$\begin{array}{l}\text { Field } \\
\text { Capacity }\end{array}$}} & & \multirow{3}{*}{$\begin{array}{l}0.124 \\
\text { c }\end{array}$} & \multirow{3}{*}{$\begin{array}{l}0.161 \\
\mathrm{~b}\end{array}$} & \multirow{3}{*}{$\begin{array}{l}0.214 \\
\mathrm{a}\end{array}$} & \multirow{3}{*}{ Sunflower } & 0 & \begin{tabular}{|l|}
0.098 \\
ij
\end{tabular} & $\begin{array}{l}0.137 \\
\mathrm{f}-\mathrm{j} \\
\end{array}$ & \begin{tabular}{|l}
0.210 \\
a-e
\end{tabular} \\
\hline & & & & & & & 100 & $\begin{array}{l}0.141 \\
e-j\end{array}$ & $\begin{array}{l}0.179 \\
\text { a-h }\end{array}$ & $\begin{array}{l}0.232 \\
\mathrm{ab}\end{array}$ \\
\hline & & & & & & & 200 & $\begin{array}{l}0.158 \\
c-j\end{array}$ & $\begin{array}{l}0.199 \\
\text { a-e }\end{array}$ & $\begin{array}{l}2.430 \\
\mathrm{a}\end{array}$ \\
\hline
\end{tabular}

\section{The Number of grains/ spike and grain yield}

The results of tables $(8,9)$ indicate that the numbers of grains and grain yield were significantly decreased in wheat grown under different soil water deficit $(85,60,35 \%)$. The irrigation treatment $85 \%$ gave the highest percentage compared with the level of field capacity $60 \%$ and $35 \%$ the ratio was $(9.5,34.2 \%)$ and $(7.6,24.5 \%)$ respectively. The reason is due to the volume of applied water which affects the growth characters such as leaf area, plant height, water content and pigment which are reflected in the yield and it's components. The results of this study were supported by Al-Temimi et al. (2013) [16] the drought stress reduce all yield components of wheat particularly the number of grains per spike. The results show that the presence of corn and sunflower residues decrease the value of the number of grains and grain yield compared with the control and decrease ratio that $(16.1,11.3 \%)$ and $(14.1,12.2 \%)$ respectively. This is due to the effect of the crop residues on the growth the of wheat and also adding of residues to the soil allelopathic compounds when decomposed are released to the soil that has a role in the inhibition of subsequent plants. Many researchers have recorded that corn, sorghum and sunflower residues contain allelopathic compounds such as vanillic acid, P-coumaric and Hydroxybenzoic all of which are phenolic acid dissolved in water and have inhibitory activity [41, 42]. Spraying ascorbic acid on the wheat plant at the concentration $200 \mathrm{ppm}$ surpassed compared with $100 \mathrm{ppm}$ and control the superiority ratio was $(23.1,10.3 \%)$ respectively. The positive response of wheat may be due the ascorbic acid has been an essential role in plants including, differentiation, regulation of growth, as well as metabolism [43]. This is consistent with Abdel Adeem and Ahmed (2017) [17] a study which shows that the spraying corn plants with ascorbic acid increased grain number per ear and grain yield.

The interaction between residues and field capacity significantly affected the number of grains and grain yield at field capacity $85 \%$ and with no residues.

The impact of the interaction of the three factors (residues $\times$ field capacity $\times$ ascorbic) gave the highest value without residues, field capacity $85 \%$ and concentration $200 \mathrm{ppm}$ of ASA. Regarding to interaction of four factors, the same results have been got the triple interaction in the sham-6-.

Examining the results we noted that Shame 6 was superior on cultivar IPA 99 for most of the studied characters. The difference may be due to two factors, The first factor is the genetic variation between the two cultivars. This result is consistent with Olaoye (2009) [44] that show the difference in genotypes leads to morphological, anatomical and physiological differences. The second factor is migh the due to the cultivars that are different in tolerance of allelopathic compounds, where it was found several mechanisms for the superiority of the cultivar including plant pigment, relative water content and antioxidant enzyme. 
This is consistent with Faysail and Ahmed (2014) [45] The of the superiority the wheat cultivar (Al-Ize) is cultivated in soil that contains residues of fenugreek rather than Talafer-3-.

It is necessary to choose the cultivars that resist to the allelopathic effect rather than the cultivars that showed sensitivity to those compounds.

Table (8) Effect of corn, sunflower residues and field capacity in number of grain/ spike of wheat treated with ascorbic acid

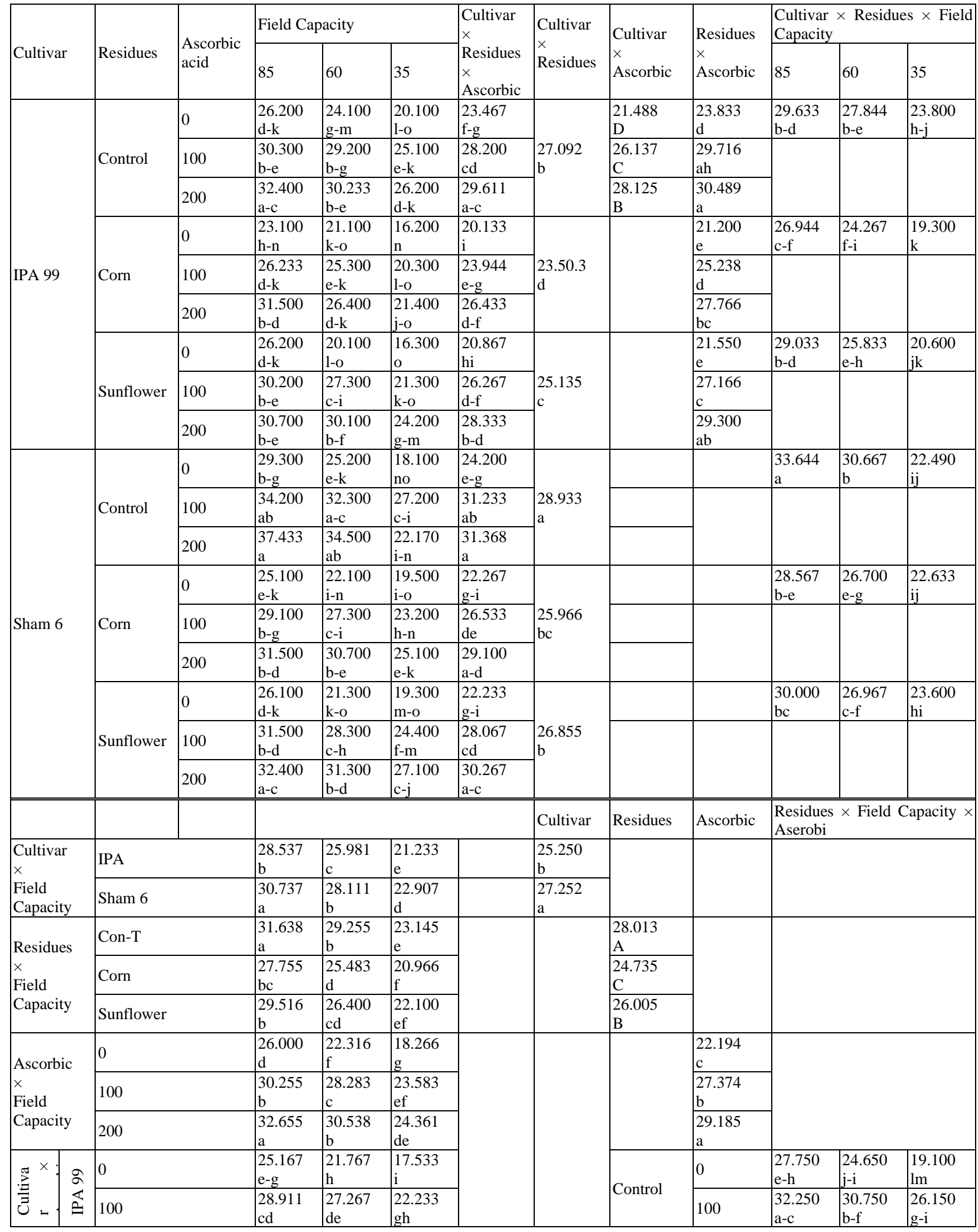


Journal of Education and Science (ISSN 1812-125X), Vol: 29, No: 2, 2020 (260-278)

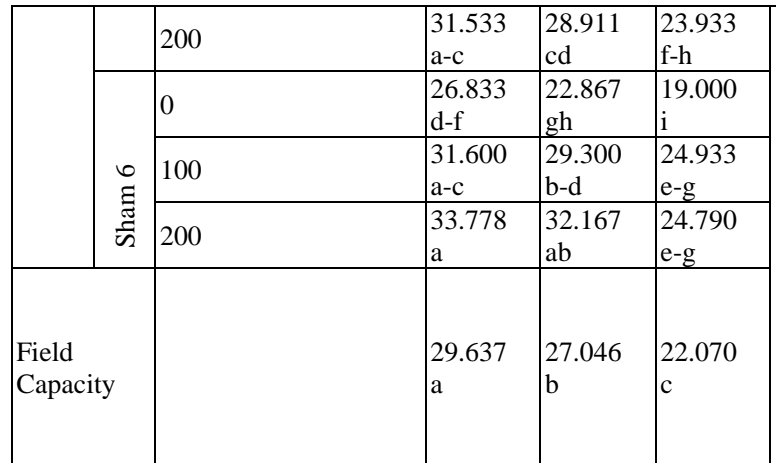

\begin{tabular}{|l|l|l|l|l|} 
& 200 & $\begin{array}{l}34.917 \\
\mathrm{a}\end{array}$ & $\begin{array}{l}32.367 \\
\mathrm{ab}\end{array}$ & $\begin{array}{l}24.185 \\
\mathrm{~h}-\mathrm{j}\end{array}$ \\
\hline \multirow{4}{*}{ Corn } & 0 & $\begin{array}{l}24.100 \\
\mathrm{~h}-\mathrm{k}\end{array}$ & $\begin{array}{l}21.600 \\
\mathrm{j}-\mathrm{l}\end{array}$ & $\begin{array}{l}17.900 \\
\mathrm{~m}\end{array}$ \\
\cline { 2 - 6 } & \multirow{2}{*}{100} & $\begin{array}{l}27.667 \\
\mathrm{f}-\mathrm{h}\end{array}$ & $\begin{array}{l}26.300 \\
\mathrm{~g}-\mathrm{i}\end{array}$ & $\begin{array}{l}21.750 \\
\mathrm{j}-\mathrm{k}\end{array}$ \\
\cline { 2 - 6 } & \multirow{2}{*}{200} & $\begin{array}{l}31.500 \\
\mathrm{a}-\mathrm{e}\end{array}$ & $\begin{array}{l}28.550 \\
\mathrm{c}-\mathrm{g}\end{array}$ & $\begin{array}{l}23.25 \\
\mathrm{i}-\mathrm{k}\end{array}$ \\
\hline \multirow{4}{*}{ Sunflower } & 0 & $\begin{array}{l}26.150 \\
\mathrm{~g}-\mathrm{i}\end{array}$ & $\begin{array}{l}20.700 \\
\mathrm{k}-\mathrm{m}\end{array}$ & $\begin{array}{l}17.800 \\
\mathrm{~m}\end{array}$ \\
\cline { 2 - 6 } & \multirow{2}{*}{100} & $\begin{array}{l}30.850 \\
\mathrm{~b}-\mathrm{f}\end{array}$ & $\begin{array}{l}27.800 \\
\mathrm{~d}-\mathrm{h}\end{array}$ & $\begin{array}{l}22.850 \\
\mathrm{i}-\mathrm{k}\end{array}$ \\
\cline { 2 - 6 } & \multirow{2}{*}{200} & $\begin{array}{l}31.550 \\
\mathrm{a}-\mathrm{d}\end{array}$ & $\begin{array}{l}30.700 \\
\mathrm{~b}-\mathrm{f}\end{array}$ & $\begin{array}{l}25.650 \\
\mathrm{~g}-\mathrm{i}\end{array}$ \\
\hline
\end{tabular}

Table (9) Effect of corn, sunflower residues and field capacity in in grain yield of wheat treated with ascorbic acid

\begin{tabular}{|c|c|c|c|c|c|c|c|c|c|c|c|c|}
\hline \multirow[b]{2}{*}{ Cultivar } & \multirow[b]{2}{*}{ Residues } & \multirow{2}{*}{$\begin{array}{l}\text { Ascorbic } \\
\text { acid }\end{array}$} & \multicolumn{3}{|c|}{ Field Capacity } & \multirow{2}{*}{$\begin{array}{l}\text { Cultivar } \\
\times \\
\text { Residues } \\
\times \\
\text { Ascorbic }\end{array}$} & \multirow{2}{*}{$\begin{array}{l}\text { Cultivar } \\
\times \\
\text { Residues }\end{array}$} & \multirow{2}{*}{$\begin{array}{l}\text { Cultivar } \\
\times \\
\text { Ascorbic }\end{array}$} & \multirow{2}{*}{$\begin{array}{l}\text { Residues } \\
\times \\
\text { Ascorbic }\end{array}$} & \multicolumn{3}{|c|}{$\begin{array}{l}\text { Cultivar } \times \text { Residues } \times \text { Field } \\
\text { Capacity }\end{array}$} \\
\hline & & & 85 & 60 & 35 & & & & & 85 & 60 & 35 \\
\hline \multirow{9}{*}{ IPA 99} & \multirow{3}{*}{ Control } & 0 & $\begin{array}{l}4.680 \\
\text { g-m }\end{array}$ & $\begin{array}{l}4.620 \\
h-m\end{array}$ & $\begin{array}{l}3.530 \\
q-s\end{array}$ & $\begin{array}{l}4.276 \\
\mathrm{~g}\end{array}$ & \multirow{3}{*}{$\begin{array}{l}4.620 \\
b c\end{array}$} & $\begin{array}{l}3.678 \\
F\end{array}$ & $\begin{array}{l}4.591 \\
\mathrm{c}\end{array}$ & $\begin{array}{l}5.000 \\
\mathrm{bc}\end{array}$ & $\begin{array}{l}4.871 \\
\mathrm{~cd}\end{array}$ & $\begin{array}{l}3.990 \\
\mathrm{gh}\end{array}$ \\
\hline & & 100 & $\begin{array}{l}4.330 \\
\text { k-o }\end{array}$ & $\begin{array}{l}4.850 \\
e-k\end{array}$ & $\begin{array}{l}4.210 \\
k-p\end{array}$ & $\begin{array}{l}4.463 \\
\mathrm{fg}\end{array}$ & & \begin{tabular}{|l|}
4.062 \\
$\mathrm{E}$
\end{tabular} & $\begin{array}{l}4.851 \\
\mathrm{~b}\end{array}$ & & & \\
\hline & & 200 & $\begin{array}{l}5.603 \\
a-d\end{array}$ & $\begin{array}{l}5.530 \\
a-d\end{array}$ & $\begin{array}{l}4.230 \\
k-p\end{array}$ & $\begin{array}{l}5.121 \\
b-d\end{array}$ & & $\begin{array}{l}4.592 \\
\mathrm{C}\end{array}$ & $\begin{array}{l}5.305 \\
\mathrm{a}\end{array}$ & & & \\
\hline & \multirow{3}{*}{ Corn } & 0 & $\begin{array}{l}3.730 \\
\mathrm{o}-\mathrm{s}\end{array}$ & $\begin{array}{l}3.140 \\
\text { st }\end{array}$ & $\begin{array}{l}2.713 \\
\mathrm{ti}\end{array}$ & $\begin{array}{l}3.194 \\
\mathrm{i}\end{array}$ & \multirow{3}{*}{$\begin{array}{l}3.778 \\
d\end{array}$} & & $\begin{array}{l}3.437 \\
\mathrm{f}\end{array}$ & $\begin{array}{l}4.223 \\
\text { fg }\end{array}$ & $\begin{array}{l}3.863 \\
h\end{array}$ & $\begin{array}{l}3.247 \\
\mathrm{i}\end{array}$ \\
\hline & & 100 & $\begin{array}{l}4.310 \\
\text { k-o }\end{array}$ & $\begin{array}{l}3.900 \\
n-r\end{array}$ & $\begin{array}{l}.410 \\
\mathrm{rs}\end{array}$ & $\begin{array}{l}3.873 \\
\mathrm{~h}\end{array}$ & & & \begin{tabular}{|l|}
4.278 \\
$\mathrm{~d}$ \\
\end{tabular} & & & \\
\hline & & 200 & $\begin{array}{l}4.630 \\
\text { h-m }\end{array}$ & $\begin{array}{l}4.550 \\
\mathrm{i}-\mathrm{m}\end{array}$ & $\begin{array}{l}3.620 \\
p-s\end{array}$ & $\begin{array}{l}4.2667 \\
\mathrm{~g}\end{array}$ & & & \begin{tabular}{|l}
4.596 \\
$\mathrm{c}$ \\
\end{tabular} & & & \\
\hline & \multirow{3}{*}{ Sunflower } & 0 & $\begin{array}{l}4.150 \\
1-q\end{array}$ & $\begin{array}{l}3.336 \\
\text { rs }\end{array}$ & $\begin{array}{l}3.210 \\
\text { st }\end{array}$ & $\begin{array}{l}3.565 \\
h\end{array}$ & \multirow{3}{*}{$\begin{array}{l}3.935 \\
d\end{array}$} & & $\begin{array}{l}3.886 \\
\mathrm{e}\end{array}$ & $\begin{array}{l}4.643 \\
\text { de }\end{array}$ & $\begin{array}{l}3.818 \\
\mathrm{~h}\end{array}$ & $\begin{array}{l}3.343 \\
\mathrm{i}\end{array}$ \\
\hline & & 100 & $\begin{array}{l}4.730 \\
\mathrm{f}-\mathrm{m}\end{array}$ & $\begin{array}{l}3.280 \\
\mathrm{v}-\mathrm{t}\end{array}$ & $\begin{array}{l}3.540 \\
q-s\end{array}$ & $\begin{array}{l}3.850 \\
h\end{array}$ & & & $\begin{array}{l}4.361 \\
\mathrm{~d}\end{array}$ & & & \\
\hline & & 200 & $\begin{array}{l}5.050 \\
d-j\end{array}$ & $\begin{array}{l}4.840 \\
\mathrm{e}-\mathrm{k}\end{array}$ & $\begin{array}{l}3.280 \\
\mathrm{r}-\mathrm{t}\end{array}$ & $\begin{array}{l}4.390 \\
\mathrm{fg}\end{array}$ & & & $\begin{array}{l}4.833 \\
\mathrm{~b}\end{array}$ & & & \\
\hline \multirow{10}{*}{ Sham 6} & \multirow{3}{*}{ Control } & 0 & $\begin{array}{l}5.210 \\
b-g\end{array}$ & $\begin{array}{l}4.780 \\
\mathrm{e}-1\end{array}$ & $\begin{array}{l}4.730 \\
f-m\end{array}$ & $\begin{array}{l}4.906 \\
c-e\end{array}$ & \multirow{3}{*}{$\begin{array}{l}5.212 \\
\mathrm{a}\end{array}$} & $\begin{array}{l}4.297 \\
D\end{array}$ & & $\begin{array}{l}5.620 \\
\mathrm{a}\end{array}$ & $\begin{array}{l}5.320 \\
\mathrm{ab}\end{array}$ & $\begin{array}{l}4.696 \\
\mathrm{~cd}\end{array}$ \\
\hline & & 100 & $\begin{array}{l}5.720 \\
\mathrm{a}-\mathrm{c}\end{array}$ & $\begin{array}{l}5.370 \\
a-f\end{array}$ & $\begin{array}{l}4.630 \\
h-m\end{array}$ & $\begin{array}{l}5.240 \\
\mathrm{a}-\mathrm{c} \\
\end{array}$ & & \begin{tabular}{|l|}
4.932 \\
$\mathrm{~B}$ \\
\end{tabular} & & & & \\
\hline & & 200 & $\begin{array}{l}5.930 \\
\mathrm{a}\end{array}$ & $\begin{array}{l}5.810 \\
a b\end{array}$ & $\begin{array}{l}4.730 \\
\mathrm{f}-\mathrm{m}\end{array}$ & $\begin{array}{l}5.490 \\
\mathrm{a}\end{array}$ & & $\begin{array}{l}5.231 \\
\mathrm{~A}\end{array}$ & & & & \\
\hline & \multirow{3}{*}{ Corn } & 0 & $\begin{array}{l}4.250 \\
k-f \\
\end{array}$ & $\begin{array}{l}3.730 \\
\mathrm{o}-\mathrm{S} \\
\end{array}$ & $\begin{array}{l}3.360 \\
\mathrm{rs}\end{array}$ & $\begin{array}{l}3.780 \\
h \\
\end{array}$ & \multirow{3}{*}{$\begin{array}{l}4.463 \\
\mathrm{c}\end{array}$} & & & $\begin{array}{l}4.835 \\
\text { cd }\end{array}$ & $\begin{array}{l}4.554 \\
\text { de }\end{array}$ & $\begin{array}{l}4.000 \\
\mathrm{gh}\end{array}$ \\
\hline & & 100 & $\begin{array}{l}5.010 \\
d-j \\
\end{array}$ & $\begin{array}{l}4.830 \\
\text { e-k }\end{array}$ & $\begin{array}{l}4.210 \\
\mathrm{k}-\mathrm{p}\end{array}$ & $\begin{array}{l}4.683 \\
\text { ef }\end{array}$ & & & & & & \\
\hline & & 200 & $\begin{array}{l}5.246 \\
\text { b-g }\end{array}$ & $\begin{array}{l}5.103 \\
\mathrm{c}-\mathrm{i}\end{array}$ & $\begin{array}{l}4.430 \\
\mathrm{j}-\mathrm{n}\end{array}$ & $\begin{array}{l}4.926 \\
\mathrm{c}-\mathrm{e}\end{array}$ & & & & & & \\
\hline & \multirow{3}{*}{ Sunflower } & 0 & $\begin{array}{l}4.660 \\
h-m\end{array}$ & $\begin{array}{l}4.100 \\
\mathrm{~m}-\mathrm{q}\end{array}$ & $\begin{array}{l}3.860 \\
n-r\end{array}$ & $\begin{array}{l}4.206 \\
\mathrm{~g}\end{array}$ & \multirow{3}{*}{4.785} & & & $\begin{array}{l}5.230 \\
b\end{array}$ & $\begin{array}{l}4.780 \\
\mathrm{~cd}\end{array}$ & $\begin{array}{l}4.346 \\
\text { ef }\end{array}$ \\
\hline & & 100 & $\begin{array}{l}5.320 \\
\mathrm{a}-\mathrm{g}\end{array}$ & $\begin{array}{l}4.830 \\
\mathrm{e}-\mathrm{k}\end{array}$ & $\begin{array}{l}4.470 \\
\text { i-n }\end{array}$ & $\begin{array}{l}4.873 \\
\mathrm{de}\end{array}$ & & & & & & \\
\hline & & 200 & $\begin{array}{l}5.710 \\
\mathrm{a}-\mathrm{c}\end{array}$ & $\begin{array}{l}5.410 \\
\mathrm{a}-\mathrm{e}\end{array}$ & $\begin{array}{l}4.710 \\
\mathrm{~g}-\mathrm{m}\end{array}$ & $\begin{array}{l}5.276 \\
\mathrm{ab}\end{array}$ & & & & & & \\
\hline & & & & & & & Cultivar & Residues & Ascorbic & $\begin{array}{l}\text { Residu } \\
\text { Aserob }\end{array}$ & $\times$ Field & Capacity $\times$ \\
\hline \multirow{2}{*}{$\begin{array}{l}\text { Cultivar } \\
\times \\
\text { Field } \\
\text { Capacity }\end{array}$} & \multicolumn{2}{|l|}{ IPA } & $\begin{array}{l}4.579 \\
\mathrm{c}\end{array}$ & $\begin{array}{l}4.227 \\
\mathrm{~d}\end{array}$ & $\begin{array}{l}3.527 \\
\mathrm{e}\end{array}$ & & $\begin{array}{l}4.111 \\
\mathrm{~b}\end{array}$ & & & & & \\
\hline & \multicolumn{2}{|l|}{ Sham 6} & $\begin{array}{l}5.228 \\
\mathrm{a} \\
\end{array}$ & $\begin{array}{l}4.884 \\
\mathrm{~b}\end{array}$ & $\begin{array}{l}4.347 \\
\mathrm{~d}\end{array}$ & & $\begin{array}{l}4.820 \\
\mathrm{a} \\
\end{array}$ & & & & & \\
\hline \multirow{3}{*}{$\begin{array}{l}\text { Residues } \\
\times \\
\text { Field } \\
\text { Capacity }\end{array}$} & \multicolumn{2}{|l|}{ Con- $\mathrm{T}$} & $\begin{array}{l}5.245 \\
\mathrm{a} \\
\end{array}$ & $\begin{array}{l}5.160 \\
\mathrm{a} \\
\end{array}$ & $\begin{array}{l}4.343 \\
\mathrm{~cd}\end{array}$ & & & $\begin{array}{l}4.916 \\
\text { A } \\
\end{array}$ & & & & \\
\hline & \multicolumn{2}{|l|}{ Corn } & $\begin{array}{l}4.529 \\
\mathrm{c}\end{array}$ & $\begin{array}{l}4.208 \\
\mathrm{~d}\end{array}$ & $\begin{array}{l}3.623 \\
\mathrm{f}\end{array}$ & & & 4.120 & & & & \\
\hline & Sunflower & & $\begin{array}{l}4.936 \\
\mathrm{~b}\end{array}$ & $\begin{array}{l}4.299 \\
\mathrm{~d}\end{array}$ & $\begin{array}{l}3.845 \\
\mathrm{e}\end{array}$ & & & \begin{tabular}{|l|}
4.360 \\
$B$
\end{tabular} & & & & \\
\hline $\begin{array}{l}\text { Ascorbic } \\
\times\end{array}$ & 0 & & $\begin{array}{l}4.446 \\
\mathrm{c}\end{array}$ & $\begin{array}{l}3.951 \\
\mathrm{~d}\end{array}$ & $\begin{array}{l}3.567 \\
\mathrm{e}\end{array}$ & & & & $\begin{array}{l}3.988 \\
\mathrm{c}\end{array}$ & & & \\
\hline
\end{tabular}




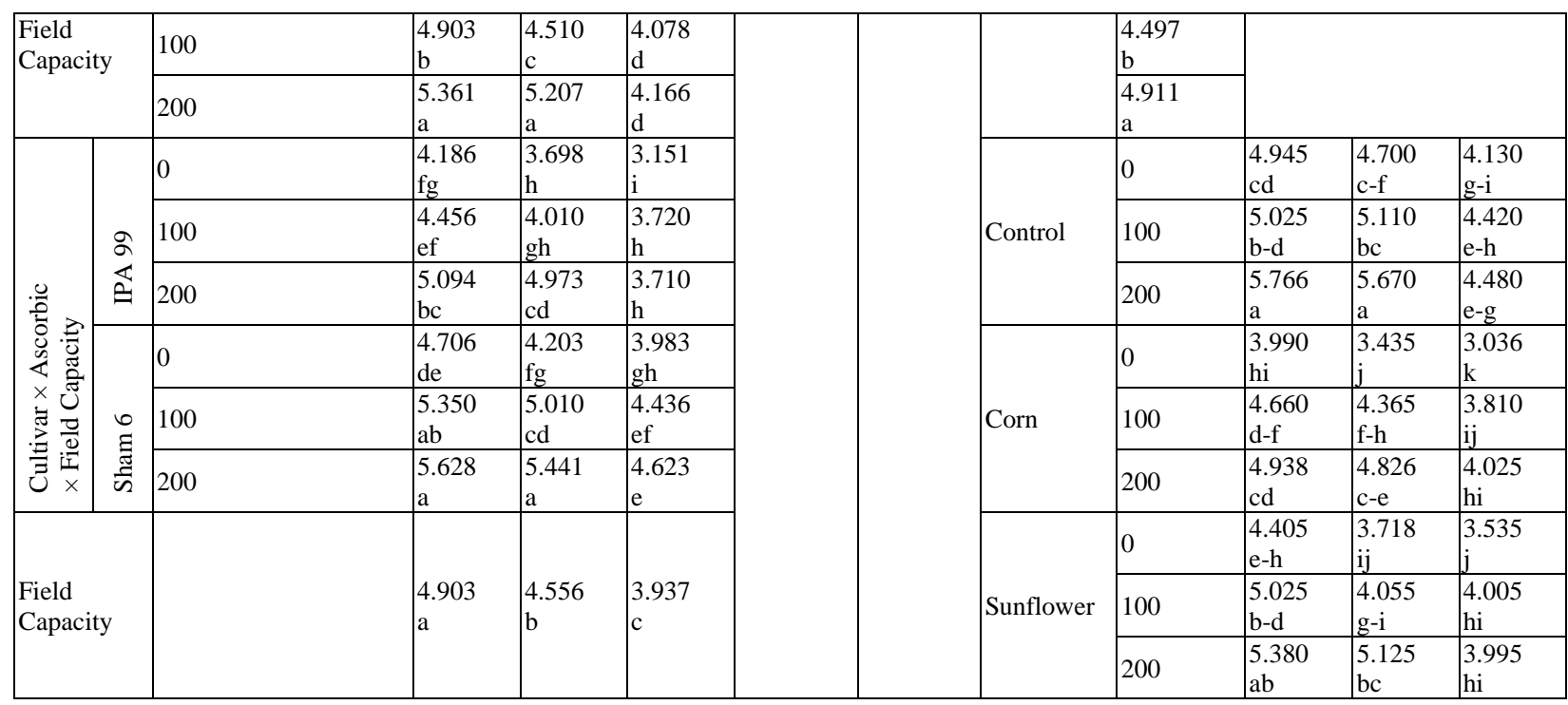

The results of the interactions for all studied factors are extended but the interaction of water stress levels and plant residues showed a more inhibitory effect than the inhibitory effect of each factor for many traits.On this side Zuo et al. (2012) [46] explained water deficit reduced plants biomass and change phenotypic characteristics such as plant height and leaf area of wheat. More then that the drought might induce the production and accumulation of most allelochemicals by the passive transport. The interaction between drought and ascorbic acid positively effected in the rate of physiological process and reduced from the negative effects of drought by improving the mentioned parameters. This is consistent in the study of Hussein and Khursheed (2014) [25] They indicate that ASA treatment alleviated the harm effect of drought by enhancing parameters (plant height, leaf area, water content and yield). Interaction between residues and ASA also reduces the inhibitory effect of residues, our current study is a pioneer in this field.

\section{Conclusion}

Plants submitted to water stress and, the addition of allelochemicals meant important stress only explainable in terms multiple stress. Sham 6 was superior at $85 \%$ field capacity, 200ppm of ASA and without residues, in most of the studied traits. Application of ASA mitigates adverse effect of environmental stresses

\section{Acknowledgments}

I would like to thank College of Education of pure Science for funding the research.

\section{Reference}

1- Central Statistical Organization. Iraq (2017).

2- Al-Fatlowe S. K. and Al-Samk K. H., J of Kerbala University., 1(2): 174-182. (2013).

3- Khaled S. A. J. plant. Physiol., 5(5): 291-294. (2010).

4- Rice E.L Allelopathy. Academic press. $2^{\text {nd }}$ New York. (1984).

5- Mohammed L. S. and Murshid, J. M., J of Kirkuk University for Agric. Sci., 9(2): 119-127. (2018).

6- Shao, H.B. Jaleel C. A. and Shao, M. A., Rev Biotechnol. 29: 131-151. (2019).

7- Mittler R., Trends in Plant. Sci., 7: 405-410. (2002).

8- Magalhaes J. P. and Church, C. M., Exp. Geronotol 41 (1): 1-15. (2006).

9- Ahmed F. F. and Morsy, M. Hd., The fifth Arabian Horti. Conference, Ismaillia, Egypt, March, 24-28. (2001). 
10- Fang Q. X. Chen, Y. H. Li, Q. Q. Yu, S. Z. Luo, Y. and Ouyang, Z. Acta Agron. Sin., 32: 861866 (2006).

11- Schon-Feid, M. A. Johnson R. C., Carver B. F. and Momhinweeg, D. W., J Crop Sci., 28: 526531. (1988).

12- Lichtenthaler H., Methods of Enzymology., 148: 350-382. (1987).

13- Goth L., Clin. Chim. Acta., 196: 143-152. (2009).

14- Kim Y. H. and Yoo T. Y., Enz. Microb. Tech., 18: 531-535. (1996).

15- Al-Obaidy B. S. J., Ph.D. Dissertation, College of Agriculture, University of Baghdad (2015). (In Arabic).

16- Al-Temimi H. N. Al-Shahwany A. W. and Al-Saadawi I. S., Iraq j of Sci., 54(3): 577-584. (2013).

17- Abdel Adeem M. S. and Ahmed M. S., J of Biotech Res. Center., 11:28-36. (2017).

18- Almahasneh H., Damascus J. for Agric Sci., 28 (2): 127-141. (2012).

19- Verma S. K. and Verma A., "A Text Book of plant physiology, Biochemistry and Biotechnology". S. Chand and Company LTD. Ram Nagar, New Delhi(2010).

20- Ali Z. A. Hussin M. M. and El-Tahar A. M., Coden (USA): IJCRGG., 8(6): 389-400. (2015).

21- Smirnoff N. and Wheeler G. L., Biochem. Mol. Biol., 35(4): 291-314. (2000).

22- Morshed J. M. and Mohammed L.S., Tikrit J for Agric Sci., 16(4): 232-247. (2016).

23- Einhelling F. A., American Chemi. Soc, Washington, DC: p 1-24. (1995).

24- Yassen A. Y., M.S.c. Thesis, College of Agriculture, University of Tikrit. (2014). (In Arabic).

25- Hussein Z. K. and Khursheed M. Q., Jordan J of Agric. Sci., 10(1): 1-15. (2014).

26- Ali F. A. M., Ali H. S. and Ali, F. H., J of Education and Sci., 17(1): 55-69. (2005).

27- Lambers H. Chapin F. S. and Pons T. L., "Plant Physiological Ecology. Spring Verlag, Berlin (1998).

28- Farooq, M. Irfan, M. Azizi, T. Ahmad, I. and Cheema S. A., J of Agron Crop Sci., 199(1) (2013).

29- Faysal M. S., Rafidian J of Science., 25(3): 26-37. (2014).

30- Darvishan M. Moghadam H. R. T. and Zahedi H., Maydica, 58(2): 195-200(2013).

31- Movani P., Agric Sci. Res. J., 1: 64-68 (2011).

32- Saker,M.T.,"Plant Physiology". Agriculture College. Mansoura University. (2010).

33- Ibrahim M. Nasser A. Zabta K. S. Asghari B. and Faizan, U., Pak. J. Bot., 45(1): 235-240. (2013).

34- Choudhury N. K. Cho, T. H. and Huffaker R. C., Plant Physioloy., 141: 551-556. (1993).

35- Grata, P. L. Polle A. Lea P. J. and Azevedo, R. A., Functional plant Bio: 32: 481-494. (2005).

36- Sharifi P. Amirnia, R. Hadi H. Majidi E. Nakoda B., Moradi M. Ronstaii and Alipoor H. M. African. J. Microbiol. Res., 6(3): 617-623. (2012).

37- Mousi A. N. and Ibrahim N. A., J of Kerbala University., 16(2): 204-216. (2018).

38- Peng S. L. Wen J. and Guo, Q. F., Acta Bot Sinica., 46: 757-766. (2004).

39- Behairy, R. T. El-Danasor, M. and Craker, L., J. of Medicinally Active Plants., 1(3): 106-112. (2012).

40- Alscher R. G. Erturk N. and Heath L. S., J. of Exp. Bot., 53: 133-141. (2002).

41- Al-Khalidi R. S. and Dawood W. M., Diyala J of Agric Sci., 9(2): 213-221. (2017).

42- Anjum T. and Bajwa R., Phytochemistry., 66: 1919-1921. (2005).

43- Horemans N. Foyer C. H. and Asard G. H., Plant Physiology., 38: 531-540. (2000).

44- Olaoye G. Bello O. B. Abubaker A. Y. Olayiwolo L. S. and Adesina O. A., Afric. J. Biol. 8(14): 3229-3238. (2009).

45- Faysail M. S. and Ahmed A. I., Res. of Reviews in Bio Sciences., 8(12): 481-489(2014).

46- Zuo S. Jinhu Z. Hongb. S. and Guangchao., African J. of Biotechnology., 9(33) 5430-5440. (2012). 